\title{
Quenched Point-to-Point Free Energy for Random Walks in Random Potentials
}

\author{
Firas Rassoul-Agha · Timo Seppäläinen
}

\begin{abstract}
We consider a random walk in a random potential on a square lattice of arbitrary dimension. The potential is a function of an ergodic environment and some steps of the walk. The potential can be unbounded, but it is subject to a moment assumption whose strictness is tied to the mixing of the environment, the best case being the i.i.d. environment. We prove that the infinite volume quenched point-to-point free energy exists and has a variational formula in terms of an entropy. We establish regularity properties of the point-to-point free energy, as a function of the potential and as a function on the convex hull of the admissible steps of the walk, and link it to the infinite volume free energy and quenched large deviations of the endpoint of the walk. One corollary is a quenched large deviation principle for random walk in an ergodic random environment, with a continuous rate function.
\end{abstract}

Keywords point-to-point $\cdot$ quenched $\cdot$ free energy $\cdot$ large deviation $\cdot$ random

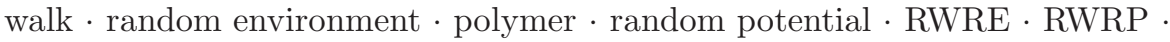
directed polymer $\cdot$ stretched polymer $\cdot$ entropy $\cdot$ variational formula

Mathematics Subject Classification (2010) 60F10 - 60K35 - 60K37 . $82 \mathrm{D} 60 \cdot 82 \mathrm{~B} 41$

\section{Introduction}

Fix a dimension $d \in \mathbb{N}$. Let $\mathcal{R} \subset \mathbb{Z}^{d}$ be a finite subset of the square lattice and let $P$ denote the distribution of the random walk on $\mathbb{Z}^{d}$ started at 0 and whose

F. Rassoul-Agha

Mathematics Department, University of Utah, 155 South 1400 East, Salt Lake City, UT 84109, USA

E-mail: firas@math.utah.edu

T. Seppäläinen

Mathematics Department, University of Wisconsin-Madison, 419 Van Vleck Hall, Madison, WI 53706, USA

E-mail: seppalai@math.wisc.edu 
transition probability is $\hat{p}_{z}=1 /|\mathcal{R}|$ for $z \in \mathcal{R}$ and $\hat{p}_{z}=0$ otherwise. In other words, the random walk picks its steps uniformly at random from $\mathcal{R}$. $E$ denotes expectation under $P$. $\mathcal{R}$ generates the additive group $\mathcal{G}=\left\{\sum_{z \in \mathcal{R}} a_{z} z: a_{z} \in\right.$ $\mathbb{Z}\}$.

An environment $\omega$ is a sample point from a probability space $(\Omega, \mathfrak{S}, \mathbb{P})$. $\Omega$ comes equipped with a group $\left\{T_{z}: z \in \mathcal{G}\right\}$ of measurable commuting transformations that satisfy $T_{x+y}=T_{x} T_{y}$ and $T_{0}$ is the identity. $\mathbb{P}$ is a $\left\{T_{z}: z \in \mathcal{G}\right\}$ invariant probability measure on $(\Omega, \mathfrak{S})$. This is summarized by the statement that $\left(\Omega, \mathfrak{S}, \mathbb{P},\left\{T_{z}: z \in \mathcal{G}\right\}\right)$ is a measurable dynamical system. For most of the time we assume that $\mathbb{P}$ is ergodic under this group, which has its usual meaning: if $A \in \mathfrak{S}$ satisfies $T_{z}^{-1} A=A$ for all $z \in \mathcal{R}$ then $\mathbb{P}(A)=0$ or 1 . For some results we also use the stronger assumption of total ergodicity which says that $\mathbb{P}(A)=0$ or 1 whenever $T_{z}^{-1} A=A$ for some extreme point $z$ of the convex hull of $\mathcal{R}$. $\mathbb{E}$ will denote expectation relative to $\mathbb{P}$.

A potential is a measurable function $g: \Omega \times \mathcal{R}^{\ell} \rightarrow \mathbb{R}$ for some integer $\ell \geq 0$. The case $\ell=0$ means that $g=g(\omega)$, a function of $\omega$ alone.

Example 1.1 (I.I.D. environment.) A natural setting is the one where $\Omega=$ $\Gamma^{\mathbb{Z}^{d}}$ is a product space with generic points $\omega=\left(\omega_{x}\right)_{x \in \mathbb{Z}^{d}}$ and translations $\left(T_{x} \omega\right)_{y}=\omega_{x+y}$, the coordinates $\omega_{x}$ are i.i.d. under $\mathbb{P}$, and $g\left(\omega, z_{1, \ell}\right)$ a local function of $\omega$, which means that $g$ depends on only finitely many coordinates $\omega_{x}$. This is a totally ergodic case. In this setting $g$ has the $r_{0^{-}}$ separated i.i.d. property for some positive integer $r_{0}$. By this we mean that if $x_{1}, \ldots, x_{m} \in \mathcal{G}$ satisfy $\left|x_{i}-x_{j}\right| \geq r_{0}$ for $i \neq j$, then the $\mathbb{R}^{\mathcal{R}^{\ell}}$-valued random vectors $\left\{\left(g\left(T_{x_{i}} \omega, z_{1, \ell}\right)\right)_{z_{1, \ell} \in \mathcal{R}^{\ell}}: 1 \leq i \leq m\right\}$ are i.i.d. under $\mathbb{P}$.

Example 1.2 (Strictly directed walk and local potential in i.i.d. environment.) A specialization of Example 1.1 where we obtain some of our best results is the case of a local potential $g$ with strictly directed paths. Strict directedness means that 0 is outside the convex hull of $\mathcal{R}$. This is equivalent to the existence of $\hat{u} \in \mathbb{Z}^{d}$ such that $\hat{u} \cdot z>0$ for all $z \in \mathcal{R}$.

Example 1.3 (Stretched polymer.) A stretched polymer has an external field $h \in \mathbb{R}^{d}$ that biases the walk, so the potential could have the form $g(\omega, z)=$ $\Psi(\omega)+h \cdot z$.

Example 1.4 (Random walk in random environment.) To cover RWRE take $\ell=1$ and $g(\omega, z)=\log p_{z}(\omega)$ where $\left(p_{z}\right)_{z \in \mathcal{R}}$ is a measurable mapping from $\Omega$ into $\mathcal{P}=\left\{\left(\rho_{z}\right)_{z \in \mathcal{R}} \in[0,1]^{\mathcal{R}}: \sum_{z} \rho_{z}=1\right\}$, the space of probability distributions on $\mathcal{R}$. The quenched path measure $Q_{0}^{\omega}$ of RWRE started at 0 is defined by the initial condition $Q_{0}^{\omega}\left(X_{0}=0\right)=1$ and the transition probability $Q_{0}^{\omega}\left(X_{n+1}=y \mid X_{n}=x\right)=p_{y-x}\left(T_{x} \omega\right)$.

Return to the general setting. Given an environment $\omega$ and an integer $n \geq 1$ define the quenched polymer measure

$$
Q_{n}^{g, \omega}(A)=\frac{1}{Z_{n}^{g, \omega}} E\left[e^{\sum_{k=0}^{n-1} g\left(T_{X_{k}} \omega, Z_{k+1, k+\ell}\right)} \mathbb{1}_{A}\left(\omega, X_{0, \infty}\right)\right],
$$


where $A$ is an event on environments and paths and

$$
Z_{n}^{g, \omega}=E\left[e^{\sum_{k=0}^{n-1} g\left(T_{X_{k}} \omega, Z_{k+1, k+\ell}\right)}\right]
$$

is the normalizing constant called the quenched partition function. This model we call random walk in a random potential (RWRP). Above $Z_{k}=X_{k}-X_{k-1}$ is a random walk step and $Z_{i, j}=\left(Z_{i}, \ldots, Z_{j}\right)$ a vector of steps. Similar notation will be used for all finite and infinite vectors and path segments, including $X_{k, \infty}=\left(X_{k}, X_{k+1}, \ldots\right)$ and $z_{1, \ell}=\left(z_{1}, \ldots, z_{\ell}\right)$ used above.

In general the measures $Q_{n}^{g, \omega}$ defined in (1.1) are not consistent as $n$ varies. But for RWRE the $\left(X_{0}, \ldots, X_{n}\right)$-marginal of $Q_{n}^{g, \omega}$ is the marginal of the quenched path measure $Q_{0}^{\omega}$.

Under some assumptions article [26] proved the $\mathbb{P}$-almost sure existence of the limit

$$
\Lambda_{\ell}(g)=\lim _{n \rightarrow \infty} n^{-1} \log E\left[e^{\sum_{k=0}^{n-1} g\left(T_{X_{k}} \omega, Z_{k+1, k+\ell}\right)}\right] .
$$

In different contexts this is called the limiting logarithmic moment generating function, the pressure, and the free energy. One of the main results of [26] was the variational characterization

$$
\Lambda_{\ell}(g)=\sup _{\mu \in \mathcal{M}_{1}\left(\boldsymbol{\Omega}_{\ell}\right), c>0}\left\{E^{\mu}[\min (g, c)]-H_{\ell}(\mu)\right\} .
$$

$\mathcal{M}_{1}\left(\boldsymbol{\Omega}_{\ell}\right)$ is the space of probability measures on $\boldsymbol{\Omega}_{\ell}=\Omega \times \mathcal{R}^{\ell}$ and $H_{\ell}(\mu)$ is a certain entropy, given in (4.6) below.

The present paper extends the entropy characterization to the quenched point-to-point free energy

$$
\Lambda_{\ell}(g, \zeta)=\lim _{n \rightarrow \infty} n^{-1} \log E\left[e^{\sum_{k=0}^{n-1} g\left(T_{X_{k}} \omega, Z_{k+1, k+\ell}\right)} \mathbb{1}\left\{X_{n}=\hat{x}_{n}(\zeta)\right\}\right]
$$

where $\zeta \in \mathbb{R}^{d}$ and $\hat{x}_{n}(\zeta)$ is a lattice point that approximates $n \zeta$. Along the way we establish regularity properties of the function $\Lambda_{\ell}(g, \zeta)$ and give another independent proof of the limit (1.2). We also relate $\Lambda_{\ell}(g)$ and $\Lambda_{\ell}(g, \zeta)$ with each other in a couple different ways, and prove a closely related large deviation principle for the distributions $Q_{n}^{g, \omega}\left\{X_{n} / n \in \cdot\right\}$ of the walk under the polymer measures $Q_{n}^{g, \omega}$. For RWRE the expectation on the right-hand side of (1.4) is the transition probability $Q_{0}^{\omega}\left\{X_{n}=\hat{x}_{n}(\zeta)\right\}$, so the limit is immediately connected to large deviations.

These results are valid for a class of unbounded potentials. When shifts of the potential are strongly mixing, it suffices to assume $g \in L^{p}$ for $p$ large enough. In particular, for an i.i.d. environment and stricly directed walks, the assumption is that $g$ is local in its dependence on $\omega$ and $g\left(\cdot, z_{1, \ell}\right) \in L^{p}(\mathbb{P})$ for some $p>d$. This, one of our main results, is reached in Theorem 4.8 in Section 4.

Literature and past results. Standard general references for RWRE are [1], [32] and [35], and for RWRP [3], [15] and [31]. Results related to ours on the point-to-point Lyapunov exponents and the quenched level $1 \mathrm{LDP}$ 
for nearest-neighbor polymers in i.i.d. random potentials have been proved by Carmona and $\mathrm{Hu}$ [2], Mourrat [21] and Zerner [36]. Some of the ideas originate in Sznitman [30] and Varadhan [33]. The Lyapunov exponents are defined by the limit

$$
\lim _{n \rightarrow \infty} n^{-1} \log E\left[e^{\sum_{k=0}^{\tau\left(\hat{x}_{n}(\zeta)\right)-1} g\left(T_{X_{k}} \omega, Z_{k+1, k+\ell}\right)} \mathbb{1}\left\{\tau\left(\hat{x}_{n}(\zeta)\right)<\infty\right\}\right],
$$

where $\tau(x)=\inf \left\{k \geq 0: X_{k}=x\right\}$.

Mourrat [21] proved a level 1 LDP for nearest-neighbor walks in an i.i.d. potential $g\left(\omega_{0}\right) \leq 0$ that permits $g=-\infty$ as long as $g\left(\omega_{x}\right)>-\infty$ percolates. In this particular case his LDP is more general than ours because we require a moment assumption on $g$. Our treatment resolves some regularity issues of the level 1 rate function raised by Carmona and $\mathrm{Hu}$ [2, Remark 1.3].

The directed i.i.d. case of Example 1.2 in dimension $d=2$, with a potential $g\left(\omega_{0}\right)$ subject to some moment assumptions, is expected to be a member of the KPZ universality class (Kardar-Parisi-Zhang). The universality conjecture is that the centered and normalized point-to-point free energy should converge to the Airy 2 process. At present such universality remains unattained. Among the lattice models studied in this paper one is currently known to be exactly solvable, namely the log-gamma polymer introduced in [28] and further studied in $[7,14]$. For that model the KPZ conjecture has been partially proved, namely the correct fluctuation exponents have been verified in some cases in [28]. Piza [22] proved in some generality that the fluctuations of the point-to-point free energy diverge at least logarithmically. KPZ universality results are further along for zero temperature polymers (oriented percolation or last-passage percolation type models). We refer the reader to [6] for a recent survey of these developments.

Organization of the paper. The existence and regularity of the quenched point-to-point free energy (1.4) and free energy (1.2) are covered in Section 2. The proof of the continuity of $\Lambda_{\ell}(g, \zeta)$ in $\zeta$ for the i.i.d. strictly directed case occupies Section 3 . In this case we also establish $L^{p}$ continuity $(p>d)$ of $\Lambda_{\ell}(g, \zeta)$ in $g$. Section 4 begins by observing the level 1 large deviation principle and the continuity of the rate function on its domain, and then develops the variational representation for the point-to-point free energy. The source for these formulas is a contraction of a higher level large deviation principle from [26].

Sections 5 and 6 discuss two examples. In Section 5 we apply the theory to a directed polymer in an i.i.d. environment in the $L^{2}$ region (a subcase of weak disorder, in dimension $d \geq 3$ ). We derive a convex-duality formula for the point-to-point free energy and identify the Markov processes that solve the variational formula. Section 6 discusses the $1+1$ dimensional log-gamma polymer, an exactly solvable model in the strong disorder regime. We take a known formula for the point-to-point free energy [14,28] and relate it to one of the variational formulas given in Section 4 of the present paper.

Notation and conventions. On a product space $\Omega=\Gamma^{\mathbb{Z}^{d}}$ with generic points $\omega=\left(\omega_{x}\right)_{x \in \mathbb{Z}^{d}}$, a local function $g(\omega)$ is a function of only finitely many 
coordinates $\omega_{x} . \mathbb{E}$ and $\mathbb{P}$ refer to the background measure on the environments $\omega$. For the set $\mathcal{R} \subset \mathbb{Z}^{d}$ of admissible steps we define $M=\max \{|z|: z \in \mathcal{R}\}$, and denote its convex hull in $\mathbb{R}^{d}$ by $\mathcal{U}=\left\{\sum_{z \in \mathcal{R}} a_{z} z: 0 \leq a_{z} \in \mathbb{R}, \sum_{z} a_{z}=1\right\}$. The steps of an admissible path $\left(x_{k}\right)$ are $z_{k}=x_{k}-x_{k-1} \in \mathcal{R}$.

In general, the convex hull of a set $\mathcal{I}$ is co $\mathcal{I}$. A convex set $\mathcal{C}$ has its relative interior ri $\mathcal{C}$, its set of extreme points ex $\mathcal{C}$, and its affine hull aff $\mathcal{C}$. The upper semicontinuous regularization of a function $f$ is denoted by $f^{\text {usc }}(x)=$ $\inf _{\text {open } B \ni x} \sup _{y \in B} f(y)$ with an analogous definition for $f^{\text {lsc }} \cdot E^{\mu}[f]=\int f d \mu$ denotes expectation under the measure $\mu$. As usual, $\mathbb{N}=\{1,2,3, \ldots\}$ and $\mathbb{Z}_{+}=\{0,1,2, \ldots\} . x \vee y=\max (x, y)$ and $x \wedge y=\min (x, y)$.

\section{Existence and regularity of the quenched point-to-point free energy}

Standing assumptions for this section are that $\left(\Omega, \mathfrak{S}, \mathbb{P},\left\{T_{z}: z \in \mathcal{G}\right\}\right)$ is a measurable dynamical system and $\mathcal{R}$ is finite. This will not be repeated in the statements of the theorems. When ergodicity is assumed it is mentioned. For the rest of this section we fix the integer $\ell \geq 0$. Define the space $\Omega_{\ell}=\Omega \times \mathcal{R}^{\ell}$. If $\ell=0$ then $\Omega_{\ell}=\Omega$. Convex analysis will be important throughout the paper. The convex hull of $\mathcal{R}$ is denoted by $\mathcal{U}$, the set of extreme points of $\mathcal{U}$ is $\operatorname{ex} \mathcal{U} \subset \mathcal{R}$, and ri $\mathcal{U}$ is the relative interior of $\mathcal{U}$.

The following is our key assumption.

Definition 2.1 Let $\ell \in \mathbb{Z}_{+}$. A function $g: \boldsymbol{\Omega}_{\ell} \rightarrow \mathbb{R}$ is in class $\mathcal{L}$ if for each $\tilde{z}_{1, \ell} \in \mathcal{R}^{\ell}$ these properties hold: $g\left(\cdot, \tilde{z}_{1, \ell}\right) \in L^{1}(\mathbb{P})$ and for any nonzero $z \in \mathcal{R}$

$$
\varlimsup_{\varepsilon \searrow 0} \varlimsup_{n \rightarrow \infty} \max _{x \in \mathcal{G}:|x| \leq n} \frac{1}{n} \sum_{0 \leq k \leq \varepsilon n}\left|g\left(T_{x+k z} \omega, \tilde{z}_{1, \ell}\right)\right|=0 \quad \text { for } \mathbb{P} \text {-a.e. } \omega \text {. }
$$

Membership $g \in \mathcal{L}$ depends on a combination of mixing properties of $\mathbb{P}$ and moment properties of $g$. If $\mathbb{P}$ is an arbitrary ergodic measure then in general we must assume $g$ bounded to guarantee $g \in \mathcal{L}$, except that if $d=1$ then $g \in L^{1}(\mathbb{P})$ is enough. Strong mixing of the process $\left\{g \circ T_{x}: x \in \mathcal{G}\right\}$ and $g \in L^{p}(\mathbb{P})$ for some large enough $p$ also guarantee $g \in \mathcal{L}$. For example, with exponential mixing $p>d$ is enough. This is the case in particular if $g$ has the $r_{0}$-separated i.i.d. property mentioned in Example 1.1. Lemma A.4 of [26] gives a precise statement.

We now define the lattice points $\hat{x}_{n}(\zeta)$ that appear in the point-to-point free energy (1.4). For each point $\zeta \in \mathcal{U}$ fix weights $\beta_{z}(\zeta) \in[0,1]$ such that $\sum_{z \in \mathcal{R}} \beta_{z}(\zeta)=1$ and $\zeta=\sum_{z \in \mathcal{R}} \beta_{z}(\zeta) z$. Then define a path

$$
\hat{x}_{n}(\zeta)=\sum_{z \in \mathcal{R}}\left(\left\lfloor n \beta_{z}(\zeta)\right\rfloor+b_{z}^{(n)}(\zeta)\right) z, \quad n \in \mathbb{Z}_{+},
$$

where $b_{z}^{(n)}(\zeta) \in\{0,1\}$ are arbitrary but subject to these constraints: if $\beta_{z}(\zeta)=$ 0 then $b_{z}^{(n)}(\zeta)=0$, and $\sum_{z} b_{z}^{(n)}(\zeta)=n-\sum_{z \in \mathcal{R}}\left\lfloor n \beta_{z}(\zeta)\right\rfloor$. In other words, 
$\hat{x}_{n}(\zeta)$ is a lattice point that approximates $n \zeta$, is precisely $n \mathcal{R}$-steps away from the origin, and uses only those steps that appear in the particular convex representation $\zeta=\sum_{z} \beta_{z} z$ that was picked. When $\zeta \in \mathcal{U} \cap \mathbb{Q}^{d}$ we require that $\beta_{z}(\zeta)$ be rational. This is possible by Lemma A.1 of [26]. If we only cared about $\Lambda_{\ell}(g, \zeta)$ for rational $\zeta$ we could allow much more general paths, see Theorem 2.7 below.

The next theorem establishes the existence of the quenched point-to-point free energy (a) and free energy (b). Introduce the empirical measure $R_{n}^{\ell}$ by

$$
R_{n}^{\ell}(g)=n^{-1} \sum_{k=0}^{n-1} g\left(T_{X_{k}} \omega, Z_{k+1, k+\ell}\right) .
$$

Theorem 2.2 Let $g \in \mathcal{L}$.

(a) For $\mathbb{P}$-a.e. $\omega$ and simultaneously for all $\zeta \in \mathcal{U}$ the limit

$$
\Lambda_{\ell}(g, \zeta ; \omega)=\lim _{n \rightarrow \infty} n^{-1} \log E\left[e^{n R_{n}^{\ell}(g)} \mathbb{1}\left\{X_{n}=\hat{x}_{n}(\zeta)\right\}\right]
$$

exists in $(-\infty, \infty]$. For a particular $\zeta$ the limit is independent of the choice of convex representation $\zeta=\sum_{z} \beta_{z} z$ and the numbers $b_{z}^{(n)}$ that define $\hat{x}_{n}(\zeta)$ in (2.1). When $\zeta \notin \mathcal{U}$ it is natural to set $\Lambda_{\ell}(g, \zeta)=-\infty$.

(b) The limit

$$
\Lambda_{\ell}(g ; \omega)=\lim _{n \rightarrow \infty} n^{-1} \log E\left[e^{\sum_{k=0}^{n-1} g\left(T_{X_{k}} \omega, Z_{k+1, k+\ell}\right)}\right]
$$

exists $\mathbb{P}$-a.s. in $(-\infty, \infty]$ and satisfies

$$
\Lambda_{\ell}(g)=\sup _{\xi \in \mathbb{Q}^{d} \cap \mathcal{U}} \Lambda_{\ell}(g, \xi)=\sup _{\zeta \in \mathcal{U}} \Lambda_{\ell}(g, \zeta) .
$$

Formula (4.3) in Section 4 shows how to recover $\Lambda_{\ell}(g, \zeta)$ from knowing $\Lambda_{\ell}(h)$ for a broad enough class of functions $h$.

Remark 2.3 (Conditions for finiteness.) In general, we need to assume that $g$ is bounded from above to prevent the possibility that $\Lambda_{\ell}(g, \zeta)$ takes the value $+\infty$. When $g$ has the $r_{0}$-separated i.i.d. property and $0 \notin \mathcal{U}$ as in Example 1.2, the assumption $\mathbb{E}\left[|g|^{p}\right]<\infty$ for some $p>d$ guarantees that $\Lambda_{\ell}(g, \zeta)$ and $\Lambda_{\ell}(g)$ are a.s. finite (Lemma 3.1). In fact $\Lambda_{\ell}(g, \cdot)$ is either bounded or identically $+\infty$ on ri $\mathcal{U}($ Theorem $2.5(\mathrm{~d}))$.

Let us recall facts about convex sets. A face of a convex set $\mathcal{U}$ is a convex subset $\mathcal{U}_{0}$ such that every (closed) line segment in $\mathcal{U}$ with a relative interior point in $\mathcal{U}_{0}$ has both endpoints in $\mathcal{U}_{0} . \mathcal{U}$ itself is a face. By Corollary 18.1.3 of [27] any other face of $\mathcal{U}$ is entirely contained in the relative boundary of $\mathcal{U}$. Extreme points of $\mathcal{U}$ are the zero-dimensional faces. By Theorem 18.2 of [27] each point $\zeta \in \mathcal{U}$ has a unique face $\mathcal{U}_{0}$ such that $\zeta \in \operatorname{ri} \mathcal{U}_{0}$. (An extreme case of this is $\zeta \in \operatorname{ex} \mathcal{U}$ in which case $\{\zeta\}=\mathcal{U}_{0}=$ ri $\mathcal{U}_{0}$. Note that the relative interior of a nonempty convex set is never empty.) By Theorem 18.1 of [27] if $\zeta \in \mathcal{U}$ belongs to a face $\mathcal{U}_{0}$ then any representation of $\zeta$ as a convex combination of 
elements of $\mathcal{U}$ involves only elements of $\mathcal{U}_{0}$. Lastly, Theorem 18.3 in [27] says that a face $\mathcal{U}_{0}$ is the convex hull of $\mathcal{R}_{0}=\mathcal{R} \cap \mathcal{U}_{0}$.

We address basic properties of $\Lambda_{\ell}(g, \zeta ; \omega)$. The first issue is whether it is random (genuinely a function of $\omega$ ) or deterministic (there is a value $\Lambda_{\ell}(g, \zeta)$ such that $\Lambda_{\ell}(g, \zeta ; \omega)=\Lambda_{\ell}(g, \zeta)$ for $\mathbb{P}$-almost every $\left.\omega\right)$. This will depend on the setting. If $0 \in \operatorname{ex} \mathcal{U}$ then the condition $X_{n}=0$ does not permit the walk to move and $\Lambda_{\ell}(g, 0 ; \omega)=-\log |\mathcal{R}|+g(\omega,(0, \ldots, 0))$. But even if the origin does not cause problems, $\Lambda_{\ell}(g, \zeta ; \omega)$ is not necessarily deterministic on all of $\mathcal{U}$ if $\mathbb{P}$ is not totally ergodic. For example, if $0 \neq z \in \operatorname{ex} \mathcal{U}$ then $X_{n}=n z$ is possible only by repetition of step $z$ and $\Lambda_{\ell}(g, z ; \omega)=-\log |\mathcal{R}|+\mathbb{E}\left[g(\omega,(z, \ldots, z)) \mid \mathfrak{I}_{z}\right]$, where $\mathfrak{I}_{z}$ is the $\sigma$-algebra invariant under $T_{z}$.

Theorem 2.4 Let $\mathcal{U}_{0}$ be any face of $\mathcal{U}$, possibly $\mathcal{U}$ itself. If $\mathbb{P}$ is ergodic under $\left\{T_{z}: z \in \mathcal{R} \cap \mathcal{U}_{0}\right\}$, then $\Lambda_{\ell}(g, \zeta)$ in Theorem 2.2 is deterministic simultaneously for all $\zeta \in \operatorname{ri} \mathcal{U}_{0}$.

Note that the ergodicity assumption rules out the case $\mathcal{U}_{0}=\{0\}$, which would be a face if $0 \in \operatorname{ex} \mathcal{U}$. An important special case is the totally ergodic $\mathbb{P}$. Then $\Lambda_{\ell}(g, \zeta)$ is deterministic for all $\zeta$, except again at $\zeta=0$ if $0 \in \operatorname{ex} \mathcal{U}$.

Next regularity results for $\Lambda_{\ell}(g, \zeta)$.

Theorem 2.5 Let $g \in \mathcal{L}$ and assume $\mathbb{P}$ is ergodic.

(a) $\Lambda_{\ell}(g, \zeta)$ is convex in $g$.

(b) As a function of $\zeta, \Lambda_{\ell}(g, \zeta)$ is concave on ri $\mathcal{U}$. If $\mathbb{P}$ is totally ergodic, then $\Lambda_{\ell}(g, \zeta)$ is concave on all of $\mathcal{U}$. below.

(c) $\Lambda_{\ell}(g, \zeta)$ is lower semicontinuous in $\zeta \in \mathcal{U}$ and hence uniformly bounded

(d) $\Lambda_{\ell}(g)$ is deterministic.

(e) $\Lambda_{\ell}(g, \zeta)$ is either bounded on $\mathcal{U}$, or identically $+\infty$ on ri $\mathcal{U}$. It is bounded if, and only if, $\Lambda_{\ell}(g)<\infty$.

(f) When $\Lambda_{\ell}(g)<\infty, \Lambda_{\ell}(g, \cdot)$ is continuous on ri $\mathcal{U}$ and its upper semicontinuous regularization is the same as its unique extension to a continuous function on $\mathcal{U}$.

Precisely speaking, the proof of part (b) of the theorem gives the following statement. If $\mathcal{U}_{0}$ is any face of $\mathcal{U}$ such that $\mathbb{P}$ is ergodic under $\left\{T_{z}: z \in \mathcal{U}_{0} \cap \mathcal{R}\right\}$, then $\mathbb{P}$-a.s.

$$
\Lambda_{\ell}(g, t \zeta+(1-t) \eta) \geq t \Lambda_{\ell}(g, \zeta)+(1-t) \Lambda_{\ell}(g, \eta)
$$

for all $\zeta \in \operatorname{ri} \mathcal{U}_{0}, \eta \in \mathcal{U}$ and $t \in[0,1]$.

As observed above, if $0 \in \operatorname{ex} \mathcal{U}$, then $\Lambda_{\ell}(g, 0)$ can be random. This does not harm the concavity in part (b) in the totally ergodic case, but of course it prevents continuity in $\zeta$ up to the relative boundary of $\mathcal{U}$. The situation with continuity in $\zeta \in \mathcal{U}$ is addressed in the next theorem in the i.i.d. case.

Theorem 2.6 Let $\mathbb{P}$ be an i.i.d. product measure as described in Example 1.1 and $p>d$. Let $g: \boldsymbol{\Omega}_{\ell} \rightarrow \mathbb{R}$ be a function such that for each $z_{1, \ell} \in \mathcal{R}^{\ell}, g\left(\cdot, z_{1, \ell}\right)$ is a local function of $\omega$ and a member of $L^{p}(\mathbb{P})$. 
(a) If $0 \notin \mathcal{U}$, then $\Lambda_{\ell}(g, \zeta)$ is continuous on $\mathcal{U}$.

(b) If $0 \in \operatorname{ri} \mathcal{U}$ and $g$ is bounded above, then $\Lambda_{\ell}(g, \zeta)$ is continuous on $\mathcal{U}$.

(c) If 0 is on the relative boundary of $\mathcal{U}$ and if $g$ is bounded above, then $\Lambda_{\ell}(g, \zeta)$ is continuous on ri $\mathcal{U}$, at nonzero extreme points of $\mathcal{U}$, and at any point $\zeta$ such that the face $\mathcal{U}_{0}$ satisfying $\zeta \in$ ri $\mathcal{U}_{0}$ does not contain $\{0\}$.

In (b) and (c) $g$ needs to be bounded above since otherwise $\Lambda_{\ell}(g)=\infty$ and $\Lambda_{\ell}(g, \zeta)=\infty$ for all $\zeta \in$ ri $\mathcal{U}$.

In certain situations our proof technique can be pushed further to deal with continuity up to faces including 0 . For example, when $\mathcal{R}=\{(1,0),(0,1),(0,0)\}$ it is possible to show that $\Lambda_{\ell}(g, \zeta)$ is continuous in $\zeta \in \mathcal{U} \backslash\{0\}$.

We turn to the proofs of the theorems in this section. Recall $M=\max \{|z|$ : $z \in \mathcal{R}\}$. Let

$$
D_{n}=\left\{z_{1}+\cdots+z_{n}: z_{1, n} \in \mathcal{R}^{n}\right\}
$$

denote the set of endpoints of admissible paths of length $n$. To prove Theorem 2.2 we first treat rational points $\xi \in \mathcal{U}$. In this case we can be more liberal with the function $g$ and with the paths.

Theorem 2.7 $\operatorname{Let} g\left(\cdot, z_{1, \ell}\right) \in L^{1}(\mathbb{P})$ for each $z_{1, \ell} \in \mathcal{R}^{\ell}$. Then for $\mathbb{P}$-a.e. $\omega$ and simultaneously for all $\xi \in \mathcal{U} \cap \mathbb{Q}^{d}$ the following holds: for any path $\left\{y_{n}(\xi)\right\}_{n \in \mathbb{Z}_{+}}$ such that $y_{n}(\xi)-y_{n-1}(\xi) \in \mathcal{R}$ and for some $k \in \mathbb{N}, y_{m k}(\xi)=m k \xi$ for all $m \in \mathbb{Z}_{+}$, the limit

$$
\Lambda_{\ell}(g, \xi ; \omega)=\lim _{n \rightarrow \infty} n^{-1} \log E\left[e^{n R_{n}^{\ell}(g)} \mathbb{1}\left\{X_{n}=y_{n}(\xi)\right\}\right]
$$

exists in $(-\infty, \infty]$. For a given $\xi \in \mathcal{U} \cap \mathbb{Q}^{d}$ the limit is independent of the choice of the path $\left\{y_{n}(\xi)\right\}$ subject to the condition above.

Proof of Theorem 2.7. Fix $\xi \in \mathbb{Q}^{d} \cap \mathcal{U}$, the path $y_{n}(\xi)$, and $k$ so that $y_{m k}(\xi)=$ $m k \xi$ for all $m \in \mathbb{Z}_{+}$. By the Markov property

$$
\begin{aligned}
& \log E\left[e^{(m+n) k R_{(m+n) k}^{\ell}(g)}, X_{(m+n) k}=(m+n) k \xi\right]-2 A_{\ell}(\omega) \\
& \geq \log E\left[e^{m k R_{m k}^{\ell}(g)}, X_{m k}=m k \xi\right]-2 A_{\ell}(\omega) \\
& \quad+\log E\left[e^{n k R_{n k}^{\ell}\left(g \circ T_{m k \xi}\right)}, X_{n k}=n k \xi\right]-2 A_{\ell}\left(T_{m k \xi} \omega\right)
\end{aligned}
$$

where $T_{x}$ acts by $g \circ T_{x}\left(\omega, z_{1, \ell}\right)=g\left(T_{x} \omega, z_{1, \ell}\right)$ and the errors are covered by defining

$$
A_{\ell}(\omega)=\ell \max _{y \in \mathcal{G}:|y| \leq M \ell} \max _{z_{1, \ell} \in \mathcal{R}^{\ell}} \max _{1 \leq i \leq \ell}\left|g\left(T_{-\tilde{x}_{i}} \omega, z_{1, \ell}\right)\right| \in L^{1}(\mathbb{P})
$$

Since $g \in L^{1}(\mathbb{P})$ the random variable $-\log E\left[e^{n k R_{n k}^{\ell}(g)}, X_{n k}=n k \xi\right]+$ $2 A_{\ell}(\omega)$ is $\mathbb{P}$-integrable for each $n$. By Kingman's subadditive ergodic theorem (for example in the form in [17, Theorem 2.6, page 277])

$$
\Lambda_{\ell}(g, \xi ; \omega)=\lim _{m \rightarrow \infty} \frac{1}{m k} \log E\left[e^{m k R_{m k}^{\ell}(g)}, X_{m k}=m k \xi\right]
$$


exists in $(-\infty, \infty] \mathbb{P}$-almost surely. This limit is independent of $k$ because if $k_{1}$ and $k_{2}$ both work and give distinct limits, then the limit along the subsequence of multiples of $k_{1} k_{2}$ would not be defined. Let $\Omega_{0}$ be the full probability event on which limit (2.9) holds for all $\xi \in \mathbb{Q}^{d} \cap \mathcal{U}$ and $k \in \mathbb{N}$ such that $k \xi \in \mathbb{Z}^{d}$.

Next we extend limit (2.9) to the full sequence. Given $n$ choose $m$ so that $m k \leq n<(m+1) k$. By assumption we have admissible paths from $m k \xi$ to $y_{n}(\xi)$ and from $y_{n}(\xi)$ to $(m+1) k \xi$, so we can create inequalities by restricting the expectations to follow these path segments. For convenience let us take $k>\ell$ so that $R_{(m-1) k}^{\ell}(g)$ does not depend on the walk beyond time $m k$. Then, for all $\omega$

$$
\begin{aligned}
& \log E\left[e^{n R_{n}^{\ell}(g)}, X_{n}=y_{n}(\xi)\right] \\
& \quad \geq \log E\left[e^{(m-1) k R_{(m-1) k}^{\ell}(g)}, X_{m k}=m k \xi, X_{n}=y_{n}(\xi)\right]-A_{2 k}\left(T_{m k \xi} \omega\right) \\
& \quad \geq \log E\left[e^{(m-1) k R_{(m-1) k}^{\ell}(g)}, X_{m k}=m k \xi\right]-(n-m k) \log |\mathcal{R}|-A_{2 k}\left(T_{m k \xi} \omega\right) \\
& \quad \geq \log E\left[e^{m k R_{m k}^{\ell}(g)}, X_{m k}=m k \xi\right]-k \log |\mathcal{R}|-2 A_{2 k}\left(T_{m k \xi} \omega\right)
\end{aligned}
$$

and similarly

$$
\begin{aligned}
& \log E\left[e^{(m+1) k R_{(m+1) k}^{\ell}(g)}, X_{(m+1) k}=(m+1) k \xi\right] \\
& \quad \geq \log E\left[e^{n R_{n}^{\ell}(g)}, X_{n}=y_{n}(\xi)\right]-k \log |\mathcal{R}|-2 A_{2 k}\left(T_{m k \xi} \omega\right) .
\end{aligned}
$$

Divide by $n$ and take $n \rightarrow \infty$ in the bounds developed above. Since in general $m^{-1} Y_{m} \rightarrow 0$ a.s. for identically distributed integrable $\left\{Y_{m}\right\}$, the error terms vanish in the limit. The limit holds on the full probability subset of $\Omega_{0}$ where the errors $n^{-1} A_{2 k}\left(T_{m k \xi} \omega\right) \rightarrow 0$ for all $\xi$ and $k$. We also conclude that the limit is independent of the choice of the path $y_{n}(\xi)$. Theorem 2.7 is proved.

The next lemma will help in the proof of Theorem 2.2 and the LDP in Theorem 4.1

Lemma 2.8 Let $g \in \mathcal{L}$. Define the paths $\left\{y_{n}(\xi)\right\}$ for $\xi \in \mathbb{Q}^{d} \cap \mathcal{U}$ as in Theorem 2.7. Then for $\mathbb{P}$-a.e. $\omega$, we have the following bound for all compact $K \subset \mathbb{R}^{d}$ and $\delta>0$ :

$$
\begin{aligned}
\varlimsup_{n \rightarrow \infty} n^{-1} & \log E\left[e^{n R_{n}^{\ell}(g)} \mathbb{1}\left\{X_{n} / n \in K\right\}\right] \\
\leq & \sup _{\xi \in \mathbb{Q}^{d} \cap K_{\delta} \cap \mathcal{U}} \varlimsup_{n \rightarrow \infty} n^{-1} \log E\left[e^{n R_{n}^{\ell}(g)} \mathbb{1}\left\{X_{n}=y_{n}(\xi)\right\}\right]
\end{aligned}
$$

where $K_{\delta}=\left\{\zeta \in \mathbb{R}^{d}: \exists \zeta^{\prime} \in K\right.$ with $\left.\left|\zeta-\zeta^{\prime}\right|<\delta\right\}$

Proof Fix a nonzero $\hat{z} \in \mathcal{R}$. Fix $\varepsilon \in(0, \delta /(4 M))$ and an integer $k \geq|\mathcal{R}|(1+$ $2 \varepsilon) / \varepsilon$. There are finitely many points in $k^{-1} D_{k}$ so we can fix a single integer $b$ such that $y_{m b}(\xi)=m b \xi$ for all $m \in \mathbb{Z}_{+}$and $\xi \in k^{-1} D_{k}$. 
We construct a path from each $x \in D_{n} \cap n K$ to a multiple of a point $\xi(n, x) \in K_{\delta} \cap k^{-1} D_{k}$. Begin by writing $x=\sum_{z \in \mathcal{R}} a_{z} z$ with $a_{z} \in \mathbb{Z}_{+}$and $\sum_{z \in \mathcal{R}} a_{z}=n$. Let $m_{n}=\lceil(1+2 \varepsilon) n / k\rceil$ and $s_{z}^{(n)}=\left\lceil k a_{z} /((1+2 \varepsilon) n)\right\rceil$. Then

$$
\left(1-\frac{1}{1+2 \varepsilon}\right) n^{-1} a_{z}-\frac{1}{k} \leq n^{-1} a_{z}-k^{-1} s_{z}^{(n)} \leq\left(1-\frac{1}{1+2 \varepsilon}\right) n^{-1} a_{z} .
$$

This implies that

$$
\frac{\varepsilon}{1+2 \varepsilon} \leq 1-k^{-1} \sum_{z} s_{z}^{(n)} \leq 1-\frac{1}{1+2 \varepsilon}<\frac{\delta}{2 M}
$$

and

$$
\left|k^{-1} \sum_{z \in \mathcal{R}} s_{z}^{(n)} z-n^{-1} x\right| \leq M \sum_{z \in \mathcal{R}}\left|k^{-1} s_{z}^{(n)}-n^{-1} a_{z}\right| \leq M\left(1-\frac{1}{1+2 \varepsilon}\right)<\frac{\delta}{2} .
$$

Define a point $\xi(n, x) \in K_{\delta} \cap k^{-1} D_{k}$ by

$$
\xi(n, x)=k^{-1} \sum_{z \in \mathcal{R}} s_{z}^{(n)} z+\left(1-k^{-1} \sum_{z \in \mathcal{R}} s_{z}^{(n)}\right) \hat{z} .
$$

Since $m_{n} s_{z}^{(n)} \geq a_{z}$ for each $z \in \mathcal{R}$, the sum above describes an admissible path of $m_{n} k-n$ steps from $x$ to $m_{n} k \xi(n, x)$. For each $x \in D_{n}$ and each $n$, the number of $\hat{z}$ steps in this path is at least

$$
m_{n}\left(k-\sum_{z \in \mathcal{R}} s_{z}^{(n)}\right) \geq m_{n} k \varepsilon /(1+2 \varepsilon) \geq n \varepsilon .
$$

Next, let $\ell_{n}$ be an integer such that $\left(\ell_{n}-1\right) b<m_{n} \leq \ell_{n} b$. Repeat the steps of $k \xi(n, x)$ in $(2.13) \ell_{n} b-m_{n} \leq b$ times to go from $m_{n} k \xi(n, x)$ to $\ell_{n} k b \xi(n, x)=y_{\ell_{n} k b}(\xi(n, x))$. Thus, the total number of steps to go from $x$ to $\ell_{n} k b \xi(n, x)$ is $r_{n}=\ell_{n} k b-n$. Recall that $b$ is a function of $k$ alone. So $r_{n} \leq 3 \varepsilon n$ for $n$ large enough, depending on $k, \varepsilon$. Denote this sequence of steps by $\mathbf{u}(n, x)=\left(u_{1}, \ldots, u_{r_{n}}\right)$.

We develop an estimate. Abbreviate $\bar{g}(\omega)=\max _{z_{1, \ell} \in \mathcal{R}^{\ell}}\left|g\left(\omega, z_{1, \ell}\right)\right|$.

$$
\begin{aligned}
& \frac{1}{n} \log E\left[e^{n R_{n}^{\ell}(g)} \mathbb{1}\left\{X_{n} / n \in K\right\}\right] \\
& =\frac{1}{n} \log \sum_{x \in D_{n} \cap n K} E\left[e^{n R_{n}^{\ell}(g)}, X_{n}=x\right] \\
& \leq \max _{x \in D_{n} \cap n K} \frac{1}{n} \log E\left[e^{(n-\ell) R_{n-\ell}^{\ell}(g)}, X_{n}=x\right] \\
& \quad+\max _{x \in D_{n} \cap n K} \max _{y \in \cup_{s=0}^{\ell} D_{s}} \frac{\ell}{n} \bar{g}\left(T_{x-y} \omega\right)+\frac{C \log n}{n} \\
& \leq \max _{x \in D_{n} \cap n K} \frac{1}{n} \log E\left[e^{\ell_{n} k b R_{\ell_{n} k b}^{\ell}(g)}, X_{\ell_{n} k b}=\ell_{n} k b \xi(n, x)\right] \\
& \quad+\max _{x \in D_{n} \cap n K} \frac{1}{n} \sum_{i=1}^{r_{n}} \bar{g}\left(T_{x}+u_{1}+\cdots+u_{i} \omega\right)+\frac{r_{n}}{n} \log |\mathcal{R}| \\
& \quad+\max _{x \in D_{n} \cap n K} \max _{y \in \cup_{s=0}^{\ell} D_{s}} \frac{2 \ell}{n} \bar{g}\left(T_{x-y} \omega\right)+\frac{C \log n}{n} .
\end{aligned}
$$


As $n \rightarrow \infty$ the limsup of the term in the third-to-last line of the above display is bounded above, for all $\omega$, by

$$
(1+3 \varepsilon) \sup _{\xi \in \mathbb{Q}^{d} \cap K_{\delta} \cap \mathcal{U}} \varlimsup_{n \rightarrow \infty} n^{-1} \log E\left[e^{n R_{n}^{\ell}(g)} \mathbb{1}\left\{X_{n}=y_{n}(\xi)\right\}\right] .
$$

The proof of (2.11) is complete once we show that a.s.

$$
\begin{aligned}
& \varlimsup_{\varepsilon \rightarrow 0} \varlimsup_{n \rightarrow \infty} \max _{x \in D_{n}} \frac{1}{n} \sum_{i=1}^{r_{n}} \bar{g}\left(T_{x+u_{1}+\cdots+u_{i}} \omega\right)=0 \\
& \text { and } \quad \varlimsup_{\varepsilon \rightarrow 0} \varlimsup_{n \rightarrow \infty} \max _{x \in D_{n}} \max _{y \in \cup_{s=0}^{\ell} D_{s}} \frac{1}{n} \bar{g}\left(T_{x-y} \omega\right)=0 .
\end{aligned}
$$

To this end, observe that the order in which the steps in $\mathbf{u}(n, x)$ are arranged was so far immaterial. From (2.14) the ratio of zero steps to $\hat{z}$ steps is at most $r_{n} /(n \varepsilon) \leq 3$. Start path $\mathbf{u}(n, x)$ by alternating $\hat{z}$ steps with blocks of at most 3 zero steps, until $\hat{z}$ steps and zero steps are exhausted. After that fix an ordering $\mathcal{R} \backslash\{0, \hat{z}\}=\left\{z_{1}, z_{2}, \ldots\right\}$ and arrange the rest of the path $\mathbf{u}(n, x)$ to take first all its $z_{1}$ steps, then all its $z_{2}$ steps, and so on. This leads to the bound

$$
\sum_{i=1}^{r_{n}} \bar{g}\left(T_{x+u_{1}+\cdots+u_{i}} \omega\right) \leq 4|\mathcal{R}| \max _{y \in x+\mathbf{u}(n, x)} \max _{z \in \mathcal{R} \backslash\{0\}} \sum_{i=0}^{r_{n}} \bar{g}\left(T_{y+i z} \omega\right) .
$$

The factor 4 is for repetitions of the same $\bar{g}$-value due to zero steps. By $y \in$ $x+\mathbf{u}(n, x)$ we mean that $y$ is on the path starting from $x$ and taking steps in $\mathbf{u}(n, x)$. A similar bound develops for the second line of (2.16). Then the limits in (2.16) follow from membership in $\mathcal{L}$. The lemma is proved.

Proof of Theorem 2.2. Part (a). Having proved Theorem 2.7, the next step is to deduce the existence of $\Lambda_{\ell}(g, \zeta)$ as the limit (2.3) for irrational velocities $\zeta$, on the event of full $\mathbb{P}$-probability where $\Lambda_{\ell}(g, \xi)$ exists for all rational $\xi \in \mathcal{U}$.

Let $\zeta \in \mathcal{U}$. It comes with a convex representation $\zeta=\sum_{z \in \mathcal{R}_{0}} \beta_{z} z$ with $\beta_{z}>0$ for $z \in \mathcal{R}_{0} \subset \mathcal{R}$, and its path $\hat{x}_{\text {. }}(\zeta)$ is defined as in (2.1). Let $\delta=$ $\delta(\zeta)=\min _{z \in \mathcal{R}_{0}} \beta_{z}>0$.

We approximate $\zeta$ with rational points from co $\mathcal{R}_{0}$. Let $\varepsilon>0$ and choose $\xi=\sum_{z \in \mathcal{R}_{0}} \alpha_{z} z$ with $\alpha_{z} \in[\delta / 2,1] \cap \mathbb{Q}, \sum_{z} \alpha_{z}=1$, and $\left|\alpha_{z}-\beta_{z}\right|<\varepsilon$ for all $z \in \mathcal{R}_{0}$. Let $k \in \mathbb{N}$ be such that $k \alpha_{z} \in \mathbb{N}$ for all $z \in \mathcal{R}_{0}$. Let $m_{n}=$ $\left\lfloor k^{-1}(1+4 \varepsilon / \delta) n\right\rfloor$ and $s_{z}^{(n)}=k m_{n} \alpha_{z}-\left\lfloor n \beta_{z}\right\rfloor-b_{z}^{(n)}$. Then,

$$
s_{z}^{(n)} / n \rightarrow(1+4 \varepsilon / \delta) \alpha_{z}-\beta_{z} \geq \varepsilon>0
$$

Thus $s_{z}^{(n)} \geq 0$ for large enough $n$.

Now, starting at $\hat{x}_{n}(\zeta)$ and taking each step $z \in \mathcal{R}_{0}$ exactly $s_{z}^{(n)}$ times arrives at $k m_{n} \xi$. Denote this sequence of steps by $\left\{u_{i}\right\}_{i=1}^{r_{n}}$, with $r_{n}=k m_{n}-n \leq$ 
$(4 \varepsilon / \delta) n$. We wish to develop an estimate similar to those in (2.10) and (2.15), using again $\bar{g}(\omega)=\max _{z_{1, \ell} \in \mathcal{R}^{\ell}}\left|g\left(\omega, z_{1, \ell}\right)\right|$. Define

$$
\begin{aligned}
B(\omega, n, \varepsilon, \kappa)=\kappa|\mathcal{R}| \cdot \max _{|x| \leq \kappa n} \max _{z \in \mathcal{R} \backslash\{0\}} \sum_{i=0}^{\kappa \varepsilon n} \bar{g}\left(T_{x+i z} \omega\right) \\
+\max _{x \in D_{n}} \max _{y \in \cup_{s=0}^{\ell} D_{s}} 2 \ell \bar{g}\left(T_{x-y} \omega\right) .
\end{aligned}
$$

Then develop an upper bound:

$$
\begin{array}{r}
\log E\left[e^{k m_{n} R_{k m_{n}}^{\ell}(g)} \mathbb{1}\left\{X_{k m_{n}}=k m_{n} \xi\right\}\right] \\
\geq \log E\left[e^{n R_{n}^{\ell}(g)} \mathbb{1}\left\{X_{n}=\hat{x}_{n}(\zeta)\right\}\right]-\sum_{i=0}^{r_{n}-1} \bar{g}\left(T_{\hat{x}_{n}(\zeta)+u_{1}+\cdots+u_{i}} \omega\right) \\
\quad-\max _{y \in \cup_{s=0}^{\ell} D_{s}} 2 \ell \bar{g}\left(T_{\hat{x}_{n}(\zeta)-y} \omega\right)-(4 \varepsilon / \delta) n \log |\mathcal{R}| \\
\geq \log E\left[e^{n R_{n}^{\ell}(g)} \mathbb{1}\left\{X_{n}=\hat{x}_{n}(\zeta)\right\}\right]-B(\omega, n, \varepsilon, \kappa)-(4 \varepsilon / \delta) n \log |\mathcal{R}| .
\end{array}
$$

To get the last inequality above first order the steps of the $\left\{u_{i}\right\}$ path as was done above to go from (2.16) to (2.17). In particular, the number of zero steps needs to be controlled. If $0 \in \mathcal{R}_{0}$, pick a step $\hat{z} \in \mathcal{R}_{0} \backslash\{0\}$, and from (2.18) obtain that, for large enough $n$,

$$
\frac{s_{0}^{(n)}}{s_{\tilde{z}}^{(n)}} \leq \frac{2 n\left((1+4 \varepsilon / \delta) \alpha_{0}-\beta_{0}\right)}{n \varepsilon / 2} \leq 4\left(1+\frac{4}{\delta}\right) .
$$

Thus we can exhaust the zero steps by alternating blocks of $\lceil 4(1+4 / \delta)\rceil$ zero steps with individual $\hat{z}$ steps. Consequently in the sum on the second line of (2.19) we have a bound $c(\delta)$ on the number of repetitions of individual $\bar{g}$-values. To realize the domination by $B(\omega, n, \varepsilon, \kappa)$ on the last line of (2.19), pick $\kappa>c(\delta)$ and large enough so that $\kappa \varepsilon n \geq r_{n}$ and so that $\{|x| \leq \kappa n\}$ covers $\left\{\hat{x}_{n}(\zeta)+u_{1}+\cdots+u_{i}: 0 \leq i \leq r_{n}\right\}$.

The point of formulating the error $B(\omega, n, \varepsilon, \kappa)$ with the parameter $\kappa$ is to control all the errors in (2.19) on a single event of $\mathbb{P}$-measure 1 , simultaneously for all $\zeta \in \mathcal{U}$ and countably many $\varepsilon \searrow 0$, with a choice of rational $\xi$ for each pair $(\zeta, \varepsilon)$. From $g \in \mathcal{L}$ follows that $\mathbb{P}$-a.s.

$$
\varlimsup_{\varepsilon \searrow 0} \varlimsup_{n \rightarrow \infty} n^{-1} B(\omega, n, \varepsilon, \kappa)=0 \quad \text { simultaneously for all } \kappa \in \mathbb{N} .
$$

A similar argument, with $\bar{m}_{n}=\left\lfloor k^{-1}(1-4 \varepsilon / \delta) n\right\rfloor$ and $\bar{s}_{z}^{(n)}=\left\lfloor n \beta_{z}\right\rfloor+$ $b_{z}^{(n)}(\zeta)-k \bar{m}_{n} \alpha_{z}$, gives

$$
\begin{aligned}
& \log E\left[e^{k \bar{m}_{n} R_{k \bar{m}_{n}}^{\ell}(g)} \mathbb{1}\left\{X_{k \bar{m}_{n}}=k \bar{m}_{n} \xi\right\}\right] \\
& \leq \log E\left[e^{n R_{n}^{\ell}(g)} \mathbb{1}\left\{X_{n}=\hat{x}_{n}(\zeta)\right\}\right]+C \varepsilon n \log |\mathcal{R}|+B(\omega, n, \varepsilon, \kappa) .
\end{aligned}
$$

Now in (2.19) and (2.20) divide by $n$, let $n \rightarrow \infty$ and use the existence of the limit $\Lambda_{\ell}(g, \xi)$. Since $\varepsilon>0$ can be taken to zero, we have obtained 
the following. $\Lambda_{\ell}(g, \zeta)$ exists as the limit (2.3) for all $\zeta \in \mathcal{U}$ on an event of $\mathbb{P}$-probability 1 , and

$$
\Lambda_{\ell}(g, \zeta)=\lim _{\xi_{j} \rightarrow \zeta} \Lambda_{\ell}\left(g, \xi_{j}\right)
$$

whenever $\xi_{j}$ is a sequence of rational convex combinations of $\mathcal{R}_{0}$ whose coefficients converge to the coefficients $\beta_{z}$ of $\zeta$.

At this point the value $\Lambda_{\ell}(g, \zeta)$ appears to depend on the choice of the convex representation $\zeta=\sum_{z \in \mathcal{R}_{0}} \beta_{z} z$. We show that each choice gives the same value $\Lambda_{\ell}(g, \zeta)$ as a particular fixed representation. Let $\overline{\mathcal{U}}$ be the unique face containing $\zeta$ in its relative interior and $\overline{\mathcal{R}}=\mathcal{R} \cap \overline{\mathcal{U}}$. Then we can fix a convex representation $\zeta=\sum_{z \in \overline{\mathcal{R}}} \bar{\beta}_{z} z$ with $\bar{\beta}_{z}>0$ for all $z \in \overline{\mathcal{R}}$. As above, let $\xi_{n}$ be rational points from co $\mathcal{R}_{0}$ such that $\xi_{n} \rightarrow \zeta$. The fact that $\zeta$ can be expressed as a convex combination of $\mathcal{R}_{0}$ forces $\mathcal{R}_{0} \subset \overline{\mathcal{U}}$, and consequently $\xi_{n} \in \overline{\mathcal{U}}$. By Lemma A.1, there are two rational convex representations $\xi_{n}=$ $\sum_{z \in \mathcal{R}_{0}} \alpha_{z}^{n} z=\sum_{z \in \overline{\mathcal{R}}} \bar{\alpha}_{z}^{n} z$ with $\alpha_{z}^{n} \rightarrow \beta_{z}$ and $\bar{\alpha}_{z}^{n} \rightarrow \bar{\beta}_{z}$. By Theorem 2.7 the value $\Lambda_{\ell}\left(g, \xi_{n}\right)$ is independent of the convex representation of $\xi_{n}$. Hence the limit in (2.21) shows that representations in terms of $\mathcal{R}_{0}$ and in terms of $\overline{\mathcal{R}}$ lead to the same value $\Lambda_{\ell}(g, \zeta)$.

Part (b). With the limit (2.3) in hand, limit (2.4) and the variational formula (2.5) follow from Lemma 2.8 with $K=\mathcal{U}$. Theorem 2.2 is proved.

Next we prove the statements about the randomness of $\Lambda_{\ell}$.

Proof of Theorem 2.4 Fix a face $\mathcal{U}_{0}$ and let $\mathcal{R}_{0}=\mathcal{R} \cap \mathcal{U}_{0}$. If $\xi$ is a rational point in ri $\mathcal{U}_{0}$, we can write $\xi=\sum_{z \in \mathcal{R}_{0}} \alpha_{z} z$ with $\alpha_{z}>0$ rational for all $z \in \mathcal{R}_{0}$; this is a consequence of Lemma A.1 of [26]. Let $k \geq 1$ be an integer such that $k \alpha_{z} \in \mathbb{Z}$ for each $z$. Let $z \in \mathcal{R}_{0}$. There is a path of $k-1$ steps from $(m-1) k \xi+z$ to $m k \xi$. Proceed as was done in $(2.10)$ to reach

$$
\begin{aligned}
& \Lambda_{\ell}(g, \xi) \geq \underset{m \rightarrow \infty}{\lim } \frac{1}{m k} \log E\left[e^{m k R_{m k}^{\ell}(g)}, X_{m k}=m k \xi \mid X_{1}=z\right] \\
& \geq \underset{m \rightarrow \infty}{\lim } \frac{1}{m k} \log E\left[e^{((m-1) k+1) R_{(m-1) k+1}^{\ell}(g)},\right. \\
& \left.X_{(m-1) k+1}=(m-1) k \xi+z \mid X_{1}=z\right] \\
& =\Lambda_{\ell}(g, \xi) \circ T_{z} .
\end{aligned}
$$

Thus $\Lambda_{\ell}(g, \xi)$ is $T_{z}$-invariant for each $z \in \mathcal{R}_{0}$ so by ergodicity $\Lambda_{\ell}(g, \xi)$ is deterministic. This holds for $\mathbb{P}$-a.e. $\omega$ simultaneously for all rational $\zeta \in \operatorname{ri} \mathcal{U}_{0}$. Since $\Lambda_{\ell}(g, \cdot)$ at irrational points of ri $\mathcal{U}_{0}$ can be obtained through $(2.21)$ from its values at rational points, the claim follows for all $\zeta \in \operatorname{ri} \mathcal{U}_{0}$.

Now we prove the claims regarding the regularity of $\Lambda_{\ell}$.

Proof of Theorem 2.5 Part (a). Convexity of $\Lambda_{\ell}$ in $g$ follows from Hölder's inequality. 
Part (b). First we establish concavity for rational points in ri $\mathcal{U}$ via the Markov property. For $t \in \mathbb{Q} \cap[0,1]$ and $\xi^{\prime}, \xi^{\prime \prime} \in \mathbb{Q}^{d} \cap$ ri $\mathcal{U}$ choose $k$ so that $k t \in \mathbb{Z}_{+}, k t \xi^{\prime} \in \mathbb{Z}^{d}$, and $k(1-t) \xi^{\prime \prime} \in \mathbb{Z}^{d}$. Then, as in (2.8),

$$
\begin{aligned}
& \log E\left[e^{m k R_{m k}^{\ell}(g)}, X_{m k}=m k\left(t \xi^{\prime}+(1-t) \xi^{\prime \prime}\right)\right] \\
& \geq \log E\left[e^{m k t R_{m k t}^{\ell}(g)}, X_{m k t}=m k t \xi^{\prime}\right] \\
& \quad+\log E\left[e^{m k(1-t) R_{m k(1-t)}^{\ell}\left(g \circ T_{m k t \xi^{\prime}}\right)}, X_{m k(1-t)}=m k(1-t) \xi^{\prime \prime}\right] \\
& \quad-2 A_{\ell}\left(T_{m k t \xi^{\prime}} \omega\right) .
\end{aligned}
$$

Divide by $m k$ and let $m \rightarrow \infty$. On ri $\mathcal{U} \Lambda_{\ell}(g, \cdot)$ is deterministic (Theorem 2.4), hence the second logarithmic moment generating function on the right of $(2.22)$ converges to its limit at least in probability, hence a.s. along a subsequence. In the limit we get

$$
\Lambda_{\ell}\left(g, t \xi^{\prime}+(1-t) \xi^{\prime \prime}\right) \geq t \Lambda_{\ell}\left(g, \xi^{\prime}\right)+(1-t) \Lambda_{\ell}\left(g, \xi^{\prime \prime}\right) .
$$

Now, let $\zeta=s \zeta^{\prime}+(1-s) \zeta^{\prime \prime}, s \in(0,1)$. $\zeta^{\prime}$ is written as a convex combination of a set $\mathcal{R}_{0}^{\prime}$ with positive coefficients. Similarly for $\zeta^{\prime \prime}$. Then $\zeta$ is written in terms of $\mathcal{R}_{0}=\mathcal{R}_{0}^{\prime} \cup \mathcal{R}_{0}^{\prime \prime}$, also with positive coefficients. Pick rational $t \rightarrow s$, $\xi^{\prime}$ with coefficients in $\mathcal{R}_{0}^{\prime}$ converging to those of $\zeta^{\prime}$, and similarly for $\zeta^{\prime \prime}$. Then the coefficients of $\xi=t \xi^{\prime}+(1-t) \xi^{\prime \prime}$ in $\mathcal{R}_{0}$ converge to those of $\zeta$. Concavity of $\Lambda_{\ell}(g, \cdot)$ on rationals implies then concavity on $\mathcal{U}$, via the limit in $(2.21)$.

In the totally ergodic case Theorem 2.4 implies that $\Lambda_{\ell}(g, \zeta)$ is deterministic on all of $\mathcal{U}$, except possibly at $\zeta=0$ if this is an extreme point of $\mathcal{U}$. If 0 is among $\left\{\xi^{\prime}, \xi^{\prime \prime}\right\}$ then take $\xi^{\prime}=0$ in $(2.22)$, so that, as the limit is taken to go from (2.22) to (2.23), we can take advantage of the deterministic limit $\Lambda_{\ell}\left(g, \xi^{\prime \prime}\right)$ for the shifted term on the right of (2.22). Thus, (2.23) holds for all $\xi^{\prime}, \xi^{\prime \prime} \in \mathcal{U}$. The subsequent limit to non-rational points proceeds as above.

Part (c). Here we deal with the lower semicontinuity of $\Lambda_{\ell}(g, \zeta)$ in $\zeta \in \mathcal{U}$. Fix $\zeta$ and pick $\mathcal{U} \ni \zeta_{j} \rightarrow \zeta$ that achieves the liminf of $\Lambda_{\ell}(g, \cdot)$ at $\zeta$. Since $\mathcal{R}$ is finite, one can find a further subsequence that always stays inside the convex hull $\mathcal{U}_{0}$ of some set $\mathcal{R}_{0} \subset \mathcal{R}$ of at most $d+1$ affinely independent vectors. Then, $\zeta \in \mathcal{U}_{0}$ and we can write the convex combinations $\zeta=\sum_{z \in \mathcal{R}_{0}} \beta_{z} z$ and $\zeta_{j}=\sum_{z \in \mathcal{R}_{0}} \beta_{z}^{(j)} z$. Furthermore, as before, $\beta_{z}^{(j)} \rightarrow \beta_{z}$ as $j \rightarrow \infty$. Let $\hat{\mathcal{R}}_{0}=\left\{z \in \mathcal{R}_{0}: \beta_{z}>0\right\}$ and define $\delta=\min _{z \in \hat{\mathcal{R}}_{0}} \beta_{z}>0$.

Fix $\varepsilon \in(0, \delta / 2)$ and take $j$ large enough so that $\left|\beta_{z}^{(j)}-\beta_{z}\right|<\varepsilon$ for all $z \in \mathcal{R}_{0}$. Let $m_{n}=\lceil(1+4 \varepsilon / \delta) n\rceil$ and $s_{z}^{(n)}=\left\lfloor m_{n} \beta_{z}^{(j)}\right\rfloor+b_{z}^{(n)}\left(\zeta_{j}\right)-\left\lfloor n \beta_{z}\right\rfloor-b_{z}^{(n)}(\zeta)$ for $z \in \mathcal{R}_{0}$. (If $\beta_{z}=\beta_{z}^{(j)}=0$, then simply set $s_{z}^{(n)}=0$.) Then, for $n$ large enough, $s_{z}^{(n)} \geq 0$ for each $z \in \mathcal{R}_{0}$. Now, proceed as in the proof of (2.21), by finding a path from $\hat{x}_{n}(\zeta)$ to $\hat{x}_{m_{n}}\left(\zeta_{j}\right)$. After taking $n \rightarrow \infty, j \rightarrow \infty$, then $\varepsilon \rightarrow 0$, we arrive at

$$
\varliminf_{\mathcal{U} \ni \zeta^{\prime} \rightarrow \zeta} \Lambda_{\ell}\left(g, \zeta^{\prime}\right) \geq \Lambda_{\ell}(g, \zeta)
$$


Remark 2.9 We can see here why upper semicontinuity (and hence continuity to the boundary) may in principle not hold: constructing a path from $\zeta_{j}$ to $\zeta$ is not necessarily possible since $\zeta_{j}$ may have non-zero components on $\mathcal{R}_{0} \backslash \hat{\mathcal{R}}_{0}$.

Part (d). Lower semicontinuity implies that the supremum of $\Lambda_{\ell}(g, \zeta)$ over $\zeta \in \mathcal{U}$ is the same as that over $\zeta \in \operatorname{ri} \mathcal{U}$. (c) now follows from this and the fact that $\Lambda_{\ell}(g, \zeta)$ is deterministic in ri $\mathcal{U}$.

Part (e). If $\Lambda_{\ell}(g)=\infty$ then there exists a sequence $\zeta_{n} \in$ ri $\mathcal{U}$ such that $\Lambda_{\ell}\left(g, \zeta_{n}\right) \rightarrow \infty$. One can assume $\zeta_{n} \rightarrow \zeta \in \mathcal{U}$. Let $\zeta^{\prime}$ be any point in ri $\mathcal{U}$. Pick $t \in(0,1)$ small enough for $\zeta_{n}^{\prime \prime}=\left(\zeta^{\prime}-t \zeta_{n}\right) /(1-t)$ to be in ri $\mathcal{U}$ for $n$ large enough. Then,

$$
\Lambda_{\ell}\left(g, \zeta^{\prime}\right) \geq t \Lambda_{\ell}\left(g, \zeta_{n}\right)+(1-t) \Lambda_{\ell}\left(g, \zeta_{n}^{\prime \prime}\right)
$$

Since $\Lambda_{\ell}(g, \cdot)$ is bounded below on $\mathcal{U}$, taking $n \rightarrow \infty$ in the above display implies that $\Lambda_{\ell}\left(g, \zeta^{\prime}\right)=\infty$.

Part (f). As a finite, concave function $\Lambda_{\ell}(g, \cdot)$ is continuous on the convex open set ri $\mathcal{U}$. By $\left[27\right.$, Theorem 10.3], $\Lambda_{\ell}(g, \cdot)$ has a unique continuous extension from the relative interior to the whole of $\mathcal{U}$. To see that this agrees with the upper semicontinuous regularization, consider this general situation.

Let $f$ be a bounded lower semicontinuous function on $\mathcal{U}$ that is concave in ri $\mathcal{U}$. Let $g$ be the continuous extension of $\left.f\right|_{\text {ri } \mathcal{U}}$ and $h$ the upper semicontinuous regularization of $f$ on $\mathcal{U}$. For $x$ on the relative boundary find ri $\mathcal{U} \ni x_{n} \rightarrow x$. Then $g(x)=\lim g\left(x_{n}\right)=\lim f\left(x_{n}\right) \geq f(x)$ and so $f \leq g$ and consequently $h \leq g$. Also $g(x)=\lim g\left(x_{n}\right)=\lim f\left(x_{n}\right)=\lim h\left(x_{n}\right) \leq h(x)$ and so $g \leq h$.

\section{Proof of the continuity of the quenched point to point free energy}

The proof of Theorem 2.6 is somewhat lengthy so we separate it in its own section. This continuity argument was inspired by the treatment of the case $\mathcal{R}=\left\{e_{1}, \ldots, e_{d}\right\}$ in [20] and [13]. We begin with a lemma that gives $L^{p}$ continuity of the free energy in the potential $g$.

Lemma 3.1 Let $\mathcal{U}_{0}$ be a face of $\mathcal{U}$ (the choice $\mathcal{U}_{0}=\mathcal{U}$ is allowed), and let $\mathcal{R}_{0}=\mathcal{R} \cap \mathcal{U}_{0}$ so that $\mathcal{U}_{0}=$ co $\mathcal{R}_{0}$. Assume $0 \notin \mathcal{U}_{0}$. Then an admissible $n$-step path from 0 to a point in $n \mathcal{U}_{0}$ cannot visit the same point twice.

(a) Let $h \geq 0$ be a measurable function on $\Omega$ with the $r_{0}$-separated i.i.d. property. Then there is a constant $C=C\left(r_{0}, d, M\right)$ such that, $\mathbb{P}$-almost surely,

$$
\varlimsup_{n \rightarrow \infty} \max _{\substack{x_{0, n-1}: \\ x_{k}-x_{k-1} \in \mathcal{R}_{0}}} n^{-1} \sum_{k=0}^{n-1} h\left(T_{x_{k}} \omega\right) \leq C \int_{0}^{\infty} \mathbb{P}\{h \geq s\}^{1 / d} d s .
$$

If $h \in L^{p}(\mathbb{P})$ for some $p>d$ then the right-hand side of (3.1) is finite by Chebyshev's inequality. 
(b) Let $f, g: \Omega_{\ell} \rightarrow \mathbb{R}$ be measurable functions with the $r_{0}$-separated i.i.d. property. Then with the same constant $C$ as in (3.1)

$$
\begin{gathered}
\varlimsup_{n \rightarrow \infty} \sup _{\zeta \in \mathcal{U}_{0}} \mid n^{-1} \log E\left[e^{n R_{n}^{\ell}(f)} \mathbb{1}\left\{X_{n}=\hat{x}_{n}(\zeta)\right\}\right] \\
-n^{-1} \log E\left[e^{n R_{n}^{\ell}(g)} \mathbb{1}\left\{X_{n}=\hat{x}_{n}(\zeta)\right\}\right] \mid \\
\leq C \int_{0}^{\infty} \mathbb{P}\left\{\omega: \max _{z_{1, \ell} \in \mathcal{R}^{\ell}}\left|f\left(\omega, z_{1, \ell}\right)-g\left(\omega, z_{1, \ell}\right)\right| \geq s\right\}^{1 / d} d s .
\end{gathered}
$$

Assume additionally that $f\left(\cdot, z_{1, \ell}\right), g\left(\cdot, z_{1, \ell}\right) \in L^{p}(\mathbb{P}) \forall z_{1, \ell} \in \mathcal{R}^{\ell}$ for some $p>d$. Then $f, g \in \mathcal{L}$ and for $\zeta \in \mathcal{U}_{0}$ the limits $\Lambda_{\ell}(f, \zeta)$ and $\Lambda_{\ell}(g, \zeta)$ are finite and deterministic and satisfy

$$
\sup _{\zeta \in \mathcal{U}_{0}}\left|\Lambda_{\ell}(f, \zeta)-\Lambda_{\ell}(g, \zeta)\right| \leq C \mathbb{E}\left[\max _{z_{1, \ell} \in \mathcal{R}^{\ell}}\left|f\left(\omega, z_{1, \ell}\right)-g\left(\omega, z_{1, \ell}\right)\right|^{p}\right]
$$

Strengthen the assumptions further with $0 \notin \mathcal{U}$. Then $\Lambda_{\ell}(f)$ and $\Lambda_{\ell}(g)$ are finite and deterministic and satisfy

$$
\left|\Lambda_{\ell}(f)-\Lambda_{\ell}(g)\right| \leq C \mathbb{E}\left[\max _{z_{1, \ell} \in \mathcal{R}^{\ell}}\left|f\left(\omega, z_{1, \ell}\right)-g\left(\omega, z_{1, \ell}\right)\right|^{p}\right] .
$$

Proof If $x \in n \mathcal{U}_{0}$ and $x=\sum_{i=1}^{n} z_{i}$ gives an admissible path to $x$, then $n^{-1} x=$ $n^{-1} \sum_{i=1}^{n} z_{i}$ gives a convex representation of $n^{-1} x \in \mathcal{U}_{0}$ which then cannot use points $z \in \mathcal{R} \backslash \mathcal{R}_{0}$. By the assumption $0 \notin \mathcal{U}_{0}$, points from $\mathcal{R}_{0}$ cannot sum to 0 and consequently a loop in an $\mathcal{R}_{0}$-path is impossible.

Part (a). We can assume that $r_{0}>M=\max \{|z|: z \in \mathcal{R}\}$. We bound the quantity on the left of $(3.1)$ with a greedy lattice animal $[8,12,19]$ after a suitable coarse graining of the lattice. Let $B=\left\{0,1, \ldots, r_{0}-1\right\}^{d}$ be the cube whose copies $\left\{r_{0} y+B: y \in \mathbb{Z}^{d}\right\}$ tile the lattice. Let $\mathcal{A}_{n}$ denote the set of connected subsets $\xi$ of $\mathbb{Z}^{d}$ of size $n$ that contain the origin (lattice animals).

Since the $x_{k}$ 's are distinct,

$$
\begin{aligned}
& \sum_{k=0}^{n-1} h\left(T_{x_{k}} \omega\right)=\sum_{u \in B} \sum_{y \in \mathbb{Z}^{d}} \sum_{k=0}^{n-1} \mathbb{1}_{\left\{x_{k}=r_{0} y+u\right\}} h\left(T_{r_{0} y+u} \omega\right) \\
& \leq \sum_{u \in B} \sum_{y \in \mathbb{Z}^{d}} \mathbb{1}_{\left\{x_{0, n-1} \cap\left(r_{0} y+B\right) \neq \emptyset\right\}} h\left(T_{u+r_{0} y} \omega\right) \\
& \leq \sum_{u \in B} \max _{\xi \in \mathcal{A}_{n(d-1)}} \sum_{y \in \xi} h\left(T_{u+r_{0} y} \omega\right) .
\end{aligned}
$$

The last step works as follows. Define first a vector $y_{0, n-1} \in\left(\mathbb{Z}^{d}\right)^{n}$ from the conditions $x_{i} \in r_{0} y_{i}+B, 0 \leq i<n$. Since $r_{0}$ is larger than the maximal step size $M,\left|y_{i+1}-y_{i}\right|_{\infty} \leq 1$. Points $y_{i}$ and $y_{i+1}$ may fail to be nearest neighbors, but by filling in at most $d-1$ intermediate points we get a nearest-neighbor 
sequence. This sequence can have repetitions and can have fewer than $n(d-1)$ entries, but it is contained in some lattice animal $\xi$ of $n(d-1)$ lattice points.

We can assume that the right-hand side of (3.1) is finite. This and the fact that $\left\{h\left(T_{u+r_{0} y} \omega\right): y \in \mathbb{Z}^{d}\right\}$ are i.i.d. allows us to apply limit (1.7) of Theorem 1.1 in [19]: for a finite constant $c$ and $\mathbb{P}$-a.s.

$$
\varlimsup_{n \rightarrow \infty} \max _{\substack{x_{0}, n-1 \\ x_{k}-x_{k-1} \in \mathcal{R}_{0}}} n^{-1} \sum_{k=0}^{n-1} h\left(T_{x_{k}} \omega\right) \leq|B|(d-1) c \int_{0}^{\infty} \mathbb{P}\{h \geq s\}^{1 / d} d s .
$$

With the volume $|B|=r_{0}^{d}$ this gives (3.1).

Part (b). Write $f=g+(f-g)$ in the exponent to get an estimate, uniformly in $\zeta \in \mathcal{U}_{0}$ :

$$
\begin{aligned}
& n^{-1} \log E\left[e^{n R_{n}^{\ell}(f)} \mathbb{1}\left\{X_{n}=\hat{x}_{n}(\zeta)\right\}\right] \\
& \leq n^{-1} \log E\left[e^{n R_{n}^{\ell}(g)} \mathbb{1}\left\{X_{n}=\hat{x}_{n}(\zeta)\right\}\right] \\
& \quad+\max _{\substack{x_{0, n+\ell-1}: \\
x_{k}-x_{k-1} \in \mathcal{R}_{0}}} n^{-1} \sum_{k=0}^{n-1}\left|f\left(T_{x_{k}} \omega, z_{k+1, k+\ell}\right)-g\left(T_{x_{k}} \omega, z_{k+1, k+\ell}\right)\right| .
\end{aligned}
$$

Switch the roles of $f$ and $g$ to get a bound on the absolute difference. Apply part (a) to get (3.2).

By Lemma A.4 of [26] the $L^{p}$ assumption with $p>d$ implies that $f, g \in \mathcal{L}$. Finiteness of $\Lambda_{\ell}(f, \zeta)$ comes from (3.2) with $g=0$. Chebyshev's inequality bounds the right-hand side of (3.2) with the right-hand side of (3.3).

To get (3.4) start with (3.5) without the indicators inside the expectations and with $\mathcal{R}_{0}$ replaced by $\mathcal{R}$.

Proof of Theorem 2.6 By Lemma A.4 of [26] the $L^{p}$ assumption with $p>d$ implies that $g \in \mathcal{L}$. By Lemma 3.1 in case (a), and by the upper bound assumption in the other cases, $\Lambda_{\ell}(g)<\infty$. Thereby $\Lambda_{\ell}(g, \cdot)$ is bounded on $\mathcal{U}$ and continuous on ri $\mathcal{U}$ (Theorem 2.5). Since $\Lambda_{\ell}(g, \cdot)$ is lower semicontinuous, it suffices to prove upper semicontinuity at the relative boundary of $\mathcal{U}$.

We begin by reducing the proof to the case of a bounded $g$. We can approximate $g$ in $L^{p}$ with a bounded function. In part (a) we can apply (3.3) to $\mathcal{U}_{0}=\mathcal{U}$. Then the uniformity in $\zeta$ of (3.3) implies that it suffices to prove upper semicontinuity in the case of bounded $g$. In parts (b) and (c) $g$ is bounded above to begin with. Assume that upper semicontinuity has been proved for the bounded truncation $g_{c}=g \vee c$. Then

$$
\varlimsup_{\zeta^{\prime} \rightarrow \zeta} \Lambda_{\ell}\left(g, \zeta^{\prime}\right) \leq \varlimsup_{\zeta^{\prime} \rightarrow \zeta} \Lambda_{\ell}\left(g_{c}, \zeta^{\prime}\right) \leq \Lambda_{\ell}\left(g_{c}, \zeta\right)
$$

In cases (b) and (c) the unique face $\mathcal{U}_{0}$ that contains $\zeta$ in its relative interior does not contain 0 , and we can apply (3.3) to show that $\Lambda_{\ell}\left(g_{c}, \zeta\right)$ decreases to $\Lambda_{\ell}(g, \zeta)$ which proves upper semicontinuity for $g$. We can now assume $g$ is bounded, and by subtracting a constant we can assume $g \leq 0$. 
We only prove upper semicontinuity away from the extreme points of $\mathcal{U}$. The argument for the extreme points of $\mathcal{U}$ is an easier version of the proof.

Pick a point $\zeta$ on the boundary of $\mathcal{U}$ that is not an extreme point. Let $\mathcal{U}_{0}$ be the unique face of $\mathcal{U}$ such that $\zeta \in \operatorname{ri} \mathcal{U}_{0}$. Let $\mathcal{R}_{0}=\mathcal{R} \cap \mathcal{U}_{0}$. Then $\mathcal{U}_{0}=\operatorname{co} \mathcal{R}_{0}$ and any convex representation $\zeta=\sum_{z \in \mathcal{R}} \beta_{z} z$ of $\zeta$ can only use $z \in \mathcal{R}_{0}[27$, Theorems 18.1 and 18.3].

The theorem follows if we show that for any fixed $\delta>0$ and $\xi \in \mathbb{Q}^{d} \cap \mathcal{U}$ close enough to $\zeta$ and for $k \in \mathbb{N}$ such that $k \xi \in \mathbb{Z}^{d}$,

$$
\lim _{m \rightarrow \infty} \mathbb{P}\left\{\sum_{x_{0, m k+\ell} \in \Pi_{m k, m k \xi}} e^{m k R_{m k}^{\ell}(g)} \geq e^{m k\left(\Lambda_{\ell}(g, \zeta)+\log |\mathcal{R}|\right)+6 m k \delta}\right\}=0 .
$$

Here $\Pi_{m k, m k \xi}$ is the set of admissible paths $x_{0, m k+\ell}$ such that $x_{0}=0$ and $x_{m k}=m k \xi$. It is enough to approach $\zeta$ from outside $\mathcal{U}_{0}$ because continuity on ri $\mathcal{U}_{0}$ is guaranteed by concavity, itself a consequence of the total ergodicity (Theorem 2.5(f)) of the i.i.d. $\mathbb{P}$. Fix $\delta>0$.

Since $0 \notin \mathcal{U}_{0}$ we can find a vector $\hat{u} \in \mathbb{Z}^{d}$ such that $z \cdot \hat{u}>0$ for $z \in \mathcal{R}_{0}$.

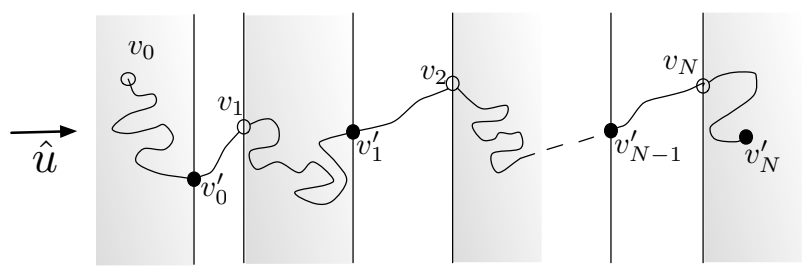

Fig. 3.1 Path segments in shaded regions are bad, the other segments are good. $v_{i}=X_{s_{i}}$ and $v_{i}^{\prime}=X_{s_{i}^{\prime}}$. Steps going up and to the right represent steps in $\mathcal{R}_{0}$.

Given a path $x_{0, m k+\ell}$ let $s_{0}=0$ and, if it exists, let $s_{0}^{\prime} \geq 0$ be its first regeneration time: this is the first time $i \in[0, m k]$ such that $x_{j} \cdot \hat{u} \leq x_{i} \cdot \hat{u}$ for $j \leq i, z_{i+1, i+\ell} \in \mathcal{R}_{0}^{\ell}$, and $x_{j} \cdot \hat{u}>x_{i+\ell} \cdot \hat{u}$ for $j \in\{i+\ell+1, \ldots, m k+\ell\}$. If $s_{0}^{\prime}$ does not exist then we set $s_{0}^{\prime}=m k+\ell$ and stop at that. Otherwise, if $s_{0}^{\prime}$ exists, then let

$$
\begin{aligned}
& s_{1}=\min \left\{j \in\left(s_{0}^{\prime}, m k+\ell\right): z_{j+1} \notin \mathcal{R}_{0}\right. \\
& \left.\quad \text { or } \exists i \in(j+1, m k+\ell] \text { such that } x_{i} \cdot \hat{u} \leq x_{j+1} \cdot \hat{u}\right\} .
\end{aligned}
$$

If such a time does not exist, then we set $s_{1}=s_{1}^{\prime}=m k+\ell$ and stop. Otherwise, define $s_{1}<s_{1}^{\prime}<s_{2}<s_{2}^{\prime}<\cdots$ inductively. Path segments $x_{s_{i}^{\prime}, s_{i+1}}$ are good and segments $x_{s_{i}, s_{i}^{\prime}}$ are bad (the paths in the gray blocks in Figure 3.1). Good segments have length at least $\ell$ and consist of only $\mathcal{R}_{0}$-steps, and distinct good segments lie in disjoint slabs (a slab is a portion of $\mathbb{Z}^{d}$ between two hyperplanes perpendicular to $\hat{u})$.

Time $m k+\ell$ may belong to an incomplete bad segment and then in the above procedure the last time defined was $s_{N}<m k+\ell$ for some $N \geq 0$ and 
we set $s_{N}^{\prime}=m k+\ell$, or to a good segment in which case the last time defined was $s_{N-1}^{\prime} \leq m k$ for some $N \geq 1$ and we set $s_{N}=s_{N}^{\prime}=m k+\ell$. There are $N$ good segments and $N+1$ bad segments, when we admit possibly degenerate first and last bad segments $x_{s_{0}, s_{0}^{\prime}}$ and $x_{s_{N}, s_{N}^{\prime}}$ (a degenerate segment has no steps). Except possibly for $x_{s_{0}, s_{0}^{\prime}}$ and $x_{s_{N}, s_{N}^{\prime}}$, each bad segment has at least one $\left(\mathcal{R} \backslash \mathcal{R}_{0}\right)$-step.

Lemma 3.2 Given $\varepsilon>0$, we can choose $\varepsilon_{0} \in(0, \varepsilon)$ such that if $|\xi-\zeta|<\varepsilon_{0}$, then the total number of steps in the bad segments in any path in $\Pi_{m k, m k \xi}$ is at most $C \varepsilon m k$ for a constant $C$. In particular, $N \leq C \varepsilon m k$.

Proof Given $\varepsilon>0$ we can find $\varepsilon_{0}>0$ such that if $|\xi-\zeta|<\varepsilon_{0}$, then any convex representation $\xi=\sum_{z \in \mathcal{R}} \alpha_{z} z$ of $\xi$ satisfies $\sum_{z \notin \mathcal{R}_{0}} \alpha_{z} \leq \varepsilon$. (Otherwise we can let $\xi \rightarrow \zeta$ and in the limit $\zeta$ would possess a convex representation with positive weight on $\mathcal{R} \backslash \mathcal{R}_{0}$.) Consequently, if $x_{0, m k+\ell} \in \Pi_{m k, m k \xi}$ and $|\xi-\zeta|<\varepsilon_{0}$ the number of $\left(\mathcal{R} \backslash \mathcal{R}_{0}\right)$-steps in $x_{0, m k+\ell}$ is bounded by $\varepsilon m k+\ell$.

Hence it is enough to show that in each bad segment, the number of $\mathcal{R}_{0^{-}}$ steps is at most a constant multiple of $\left(\mathcal{R} \backslash \mathcal{R}_{0}\right)$-steps. So consider a bad segment $x_{s_{i}, s_{i}^{\prime}}$. If $s_{i}^{\prime}=m k+\ell$ it can happen that $x_{s_{i}^{\prime}} \cdot \hat{u}<\max _{s_{i} \leq j \leq s_{i}^{\prime}} x_{j} \cdot \hat{u}$. In this case we add more steps from $\mathcal{R}_{0}$ and increase $s_{i}^{\prime}$ so that

$$
x_{s_{i}^{\prime}} \cdot \hat{u}=\max _{s_{i} \leq j \leq s_{i}^{\prime}} x_{j} \cdot \hat{u} .
$$

This only makes things worse by increasing the number of $\mathcal{R}_{0}$-steps. We proceed now by assuming (3.7).
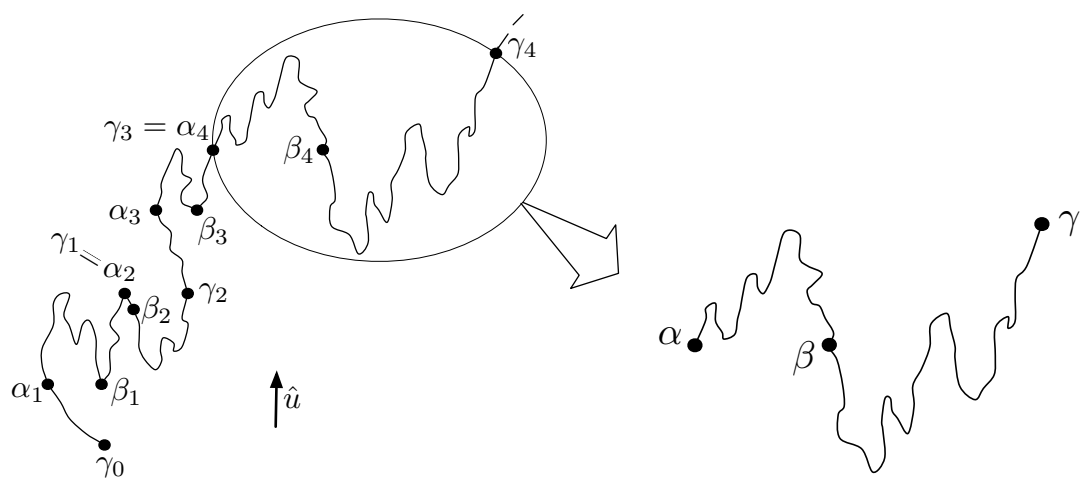

Fig. 3.2 Illustration of the stopping times $\alpha_{i}, \beta_{i}$, and $\gamma_{i}$. Note how the immediate backtracking at $\gamma_{1}$ makes $\alpha_{2}=\gamma_{1}$ and $\beta_{2}=\alpha_{2}+1$.

$$
\begin{aligned}
& \text { Start with } \gamma_{0}=s_{i} \text {. Let } \\
& \qquad \alpha_{1}=s_{i}^{\prime} \wedge \inf \left\{n \geq \gamma_{0}: \exists j>n \text { such that } x_{j} \cdot \hat{u} \leq x_{n} \cdot \hat{u}\right\} .
\end{aligned}
$$


We first control the number of $\mathcal{R}_{0}$-steps in the segment $z_{\gamma_{0}+1, \alpha_{1}}$. The segment $z_{\gamma_{0}+1, \alpha_{1}-1}$ cannot contain more than $\ell-1 \mathcal{R}_{0}$-steps in a row because any $\ell$ string of $\mathcal{R}_{0}$-steps would have begun the next good segment. Thus, the number of $\mathcal{R}_{0}$-steps in $z_{\gamma_{0}+1, \alpha_{1}}$ is bounded by $(\ell-1) \times\left(\right.$ the number of $\left(\mathcal{R} \backslash \mathcal{R}_{0}\right)$-steps) $+\ell$. Suppose $\alpha_{1}=s_{i}^{\prime}$, in other words, we already exhausted the entire bad segment. Since a bad segment contains at least one $\left(\mathcal{R} \backslash \mathcal{R}_{0}\right)$-step we are done: the number of $\mathcal{R}_{0}$-steps is bounded by $2 \ell$ times the number of $\left(\mathcal{R} \backslash \mathcal{R}_{0}\right)$-steps. So let us suppose $\alpha_{1}<s_{i}^{\prime}$ and continue with the segment $x_{\alpha_{1}, s_{i}^{\prime}}$.

Let

$$
\beta_{1}=\inf \left\{n>\alpha_{1}: x_{n} \cdot \hat{u} \leq x_{\alpha_{1}} \cdot \hat{u}\right\} \leq s_{i}^{\prime}
$$

be the time of the first backtrack after $\alpha_{1}$ and

$$
\gamma_{1}=\inf \left\{n>\beta_{1}: x_{n} \cdot \hat{u} \geq \max _{\alpha_{1} \leq j \leq \beta_{1}} x_{j} \cdot \hat{u}\right\}
$$

the time when the path gets at or above the previous maximum. Due to (3.7), $\gamma_{1} \leq s_{i}^{\prime}$.

We claim that in the segment $x_{\alpha_{1}, \gamma_{1}}$ the number of positive steps (in the $\hat{u}$-direction) is at most a constant times the number of nonpositive steps. Since $\mathcal{R}_{0}$-steps are positive steps while all nonpositive steps are $\left(\mathcal{R} \backslash \mathcal{R}_{0}\right)$-steps, this claim gives the dominance (number of $\mathcal{R}_{0}$-steps) $\leq C \times$ (number of $\left(\mathcal{R} \backslash \mathcal{R}_{0}\right)$ steps).

To see the claim, observe that at time $\beta_{1}$ the segment $x_{\alpha_{1}, \gamma_{1}}$ visits a point at or below its starting level but ends up at a new maximum level at time $\gamma_{1}$. Ignore the part of the last step $z_{\gamma_{1}}$ that takes the path above the previous maximum $\max _{\alpha_{1} \leq j \leq \beta_{1}} x_{j} \cdot \hat{u}$. Then each negative unit increment in the $\hat{u}$ direction is matched by at most two positive unit increments. Up to constant multiples, counting unit increments is the same as counting steps. (See Figure 3.2.)

Since the segment $x_{\alpha_{1}, \gamma_{1}}$ must have at least one $\left(\mathcal{R} \backslash \mathcal{R}_{0}\right)$-step, we have shown that the number of $\mathcal{R}_{0}$-steps in the segment $x_{\gamma_{0}, \gamma_{1}}$ is bounded above by $2(C \vee \ell) \times\left(\right.$ number of $\left(\mathcal{R} \backslash \mathcal{R}_{0}\right)$-steps $)$. Now repeat the previous argument, beginning at $\gamma_{1}$. Eventually the bad segment $x_{s_{i}, s_{i}^{\prime}}$ is exhausted.

Let $\mathbf{v}$ denote the collection of times $0=s_{0} \leq s_{0}^{\prime}<s_{1}<s_{1}^{\prime}<s_{2}<s_{2}^{\prime}<$ $\ldots<s_{N-1}<s_{N-1}^{\prime}<s_{N} \leq s_{N}^{\prime}=m k+\ell$, positions $v_{i}=x_{s_{i}}, v_{i}^{\prime}=x_{s_{i}^{\prime}}$, and the steps in bad path segments $u_{s_{i}, s_{i}^{\prime}}^{(i)}=z_{s_{i}+1, s_{i}^{\prime}} \cdot s_{0}=s_{0}^{\prime}$ means $u^{(0)}$ is empty.

We use the following simple fact below. There exists a function $h(\varepsilon) \searrow 0$ such that, for all $\varepsilon>0$ and $n \geq n_{0}(\varepsilon),\left(\begin{array}{c}n \\ n \varepsilon\end{array}\right) \leq e^{n h(\varepsilon)}$. (Stirling's formula shows that $n_{0}(\varepsilon)=\varepsilon^{-1}$ works.)

Lemma 3.3 With $\varepsilon>0$ fixed in Lemma 3.2, and with $m$ large enough, the number of vectors $\mathbf{v}$ is at most $C(m k)^{c_{1}} e^{m k h(\varepsilon)}$, where the function $h$ satisfies $h(\varepsilon) \rightarrow 0$ as $\varepsilon \rightarrow 0$.

Proof Recall $N \leq C \varepsilon m k$ for a constant $C$ coming from Lemma 3.2. We take $\varepsilon>0$ small enough so that $C \varepsilon<1 / 2$. A vector $\mathbf{v}$ is determined by the following choices. 
(i) The times $\left\{s_{i}, s_{i}^{\prime}\right\}_{0 \leq i \leq N}$ can be chosen in at most

$$
\sum_{N=1}^{C \varepsilon m k}\left(\begin{array}{c}
m k \\
2 N
\end{array}\right) \leq C m k\left(\begin{array}{c}
m k \\
C \varepsilon m k
\end{array}\right) \leq C m k e^{m k h(\varepsilon)} \quad \text { ways. }
$$

(ii) The steps in the bad segments, in a total of at most $|\mathcal{R}|^{C \varepsilon m k} \leq e^{m k h(\varepsilon)}$ ways.

(iii) The path increments $\left\{v_{i}-v_{i-1}^{\prime}\right\}_{1 \leq i \leq N}$ across the good segments. Their number is also bounded by $C(m k)^{c_{1}} e^{m k h(\varepsilon)}$.

The argument for (iii) is as follows. For each finite $\mathcal{R}_{0}$-increment $y \in$ $\left\{z_{1}+\cdots+z_{k}: k \in \mathbb{N}, z_{1}, \ldots, z_{k} \in \mathcal{R}_{0}\right\}$, fix a particular representation $y=\sum_{z \in \mathcal{R}_{0}} a_{z}(y) z$, identified by the vector $a(y)=\left(a_{z}(y)\right) \in \mathbb{Z}_{+}^{\mathcal{R}_{0}}$. The number of possible endpoints $\eta=\sum_{i=1}^{N}\left(v_{i}-v_{i-1}^{\prime}\right)$ is at most $C(\varepsilon m k)^{d}$ because $|m k \xi-m k \zeta|<m k \varepsilon$ and the total number of steps in all bad segments is at most $C \varepsilon m k$. Each possible endpoint $\eta$ has at most $C(m k)^{\left|\mathcal{R}_{0}\right|}$ representations $\eta=\sum_{z \in \mathcal{R}_{0}} b_{z} z$ with $\left(b_{z}\right) \in \mathbb{Z}_{+}^{\mathcal{R}_{0}}$ because projecting to $\hat{u}$ shows that each $b_{z}$ is bounded by $C m k$. Thus there are at most $C(m k)^{c_{1}}$ vectors $\left(b_{z}\right) \in \mathbb{Z}_{+}^{\mathcal{R}_{0}}$ that can represent possible endpoints of the sequence of increments. Each such vector $b=\left(b_{z}\right)$ can be decomposed into a sum of increments $b=\sum_{i=1}^{N} a^{(i)}$ in at most

$$
\prod_{z \in \mathcal{R}_{0}}\left(\begin{array}{c}
b_{z}+N \\
N
\end{array}\right) \leq\left(\begin{array}{c}
C m k+C \varepsilon m k \\
C \varepsilon m k
\end{array}\right)^{\left|\mathcal{R}_{0}\right|} \leq e^{m k h(\varepsilon)}
$$

ways. (Note that $\left(\begin{array}{c}a+b \\ b\end{array}\right)$ is increasing in both $a$ and $b$.) So all in all there are $C(m k)^{c_{1}} e^{m k h(\varepsilon)}$ possible sequences $\left\{a^{(i)}\right\}_{1 \leq i \leq N}$ of increments in the space $\mathbb{Z}_{+}^{\mathcal{R}_{0}}$ that satisfy

$$
\sum_{z \in \mathcal{R}_{0}} \sum_{i=1}^{N} a_{z}^{(i)} z=\eta \quad \text { for a possible endpoint } \eta \text {. }
$$

Map $\left\{v_{i}-v_{i-1}^{\prime}\right\}_{1 \leq i \leq N}$ to $\left\{a\left(v_{i}-v_{i-1}^{\prime}\right)\right\}_{1 \leq i \leq N}$. This mapping is $1-1$. The image is one of the previously counted sequences $\left\{a^{(i)}\right\}_{1 \leq i \leq N}$ because

$$
\sum_{z \in \mathcal{R}_{0}} \sum_{i=1}^{N} a_{z}\left(v_{i}-v_{i-1}^{\prime}\right) z=\sum_{i=1}^{N} \sum_{z \in \mathcal{R}_{0}} a_{z}\left(v_{i}-v_{i-1}^{\prime}\right) z=\sum_{i=1}^{N}\left(v_{i}-v_{i-1}^{\prime}\right)=\eta .
$$

We conclude that there are at most $C(m k)^{c_{1}} e^{m k h(\varepsilon)}$ sequences $\left\{v_{i}-v_{i-1}^{\prime}\right\}_{1 \leq i \leq N}$ of increments across the good segments. Point (iii) has been verified.

Multiplying counts (i)-(iii) proves the lemma.

Let $\Pi_{m k, m k \xi}^{\mathbf{v}}$ denote the paths in $\Pi_{m k, m k \xi}$ that are compatible with $\mathbf{v}$, that is, paths that go through space-time points $\left(x_{s_{i}}, s_{i}\right),\left(x_{s_{i}^{\prime}}, s_{i}^{\prime}\right)$ and take the specified steps in the bad segments. The remaining unspecified good segments connect $\left(x_{s_{i-1}^{\prime}}, s_{i-1}^{\prime}\right)$ to $\left(x_{s_{i}}, s_{i}\right)$ with $\mathcal{R}_{0}$-steps, for $1 \leq i \leq N$. 
Fix $\varepsilon>0$ small enough so that for large $m, C(m k)^{c_{1}} e^{m k h(\varepsilon)} \leq e^{m k \delta}$. Then our goal (3.6) follows if we show

$$
\lim _{m \rightarrow \infty} \sum_{\mathbf{v}} \mathbb{P}\left\{\sum_{x_{0, m k} \in \Pi_{m k, m k \xi}^{\mathbf{v}}} e^{m k R_{m k}^{\ell}(g)} \geq e^{m k\left(\Lambda_{\ell}(g, \zeta)+\log |\mathcal{R}|\right)+5 m k \delta}\right\}=0 .
$$

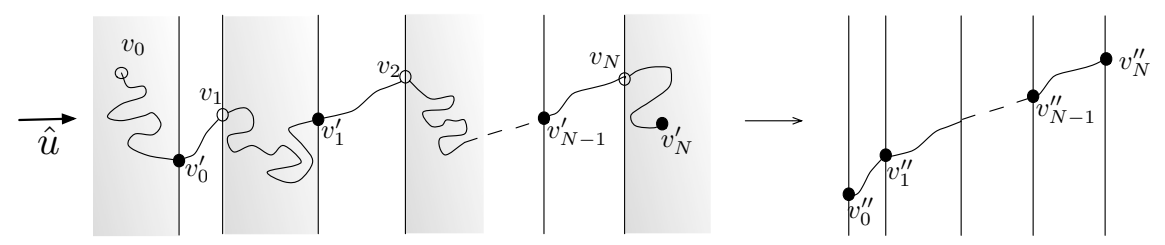

Fig. 3.3 Illustration of the construction. The shaded bad slabs of environments are deleted. The white good slabs are joined together and shifted so that the good path segments connect. So for example points $v_{1}$ and $v_{1}^{\prime}$ on the left are identified as $v_{1}^{\prime \prime}$ on the right.

Given a vector $\mathbf{v}$ and an environment $\omega$ define a new environment $\omega^{\mathbf{v}}$ by deleting the bad slabs and shifting the good slabs so that the good path increments $\left\{v_{i}-v_{i-1}^{\prime}\right\}_{1 \leq i \leq N}$ become connected. First for $x \cdot \hat{u}<0$ and $x \cdot \hat{u} \geq$ $\sum_{j=0}^{N-1}\left(v_{j+1}-v_{j}^{\prime}\right) \cdot \hat{u}$ sample $\omega_{x}^{\mathbf{v}}$ fresh (this part of space is irrelevant). For a point $x$ in between pick $i \geq 0$ such that

$$
\sum_{j=1}^{i}\left(v_{j}-v_{j-1}^{\prime}\right) \cdot \hat{u} \leq x \cdot \hat{u}<\sum_{j=1}^{i+1}\left(v_{j}-v_{j-1}^{\prime}\right) \cdot \hat{u}
$$

and put $y=\sum_{j=1}^{i}\left(v_{j}-v_{j-1}^{\prime}\right)$. Then set $\omega_{x}^{\mathbf{v}}=\omega_{v_{i}^{\prime}+x-y}$.

For a fixed $\mathbf{v}$, each path $x_{0, m k+\ell} \in \Pi_{m k, m k \xi}^{\mathbf{v}}$ is mapped in a 1-1 fashion to a new path $x_{0, \tau(\mathbf{v})+\ell-1}^{\mathbf{v}}$ as follows. Set

$$
\tau(\mathbf{v})=\sum_{j=1}^{N}\left(s_{j}-s_{j-1}^{\prime}\right)-\ell
$$

Given time point $t \in\{0, \ldots, \tau(\mathbf{v})+\ell-1\}$ pick $i \geq 0$ such that

$$
\sum_{j=1}^{i}\left(s_{j}-s_{j-1}^{\prime}\right) \leq t<\sum_{j=1}^{i+1}\left(s_{j}-s_{j-1}^{\prime}\right)
$$

Then with $s=\sum_{j=0}^{i}\left(s_{j}^{\prime}-s_{j}\right)$ and $u=\sum_{j=0}^{i}\left(v_{j}^{\prime}-v_{j}\right)$ set $x_{t}^{\mathbf{v}}=x_{t+s}-u$. This mapping of $\omega$ and $x_{0, m k+\ell}$ moves the good slabs of environments together with the good path segments so that $\omega_{x_{t}^{\mathrm{v}}}^{\mathbf{v}}=\omega_{x_{t+s}}$. (See Figure 3.3.) The sum of the good increments that appeared in Lemma 3.3 is now

$$
x_{\tau(\mathbf{v})+\ell}^{\mathbf{v}}=x_{s_{N}}-\sum_{j=0}^{N-1}\left(v_{j}^{\prime}-v_{j}\right)=v_{N}-\sum_{j=0}^{N-1}\left(v_{j}^{\prime}-v_{j}\right)=\sum_{j=1}^{N}\left(v_{j}-v_{j-1}^{\prime}\right) .
$$


Define $\eta(\mathbf{v}) \in \mathcal{U}_{0}$ by

$$
x_{\tau(\mathbf{v})}^{\mathbf{v}}=\tau(\mathbf{v}) \eta(\mathbf{v}) .
$$

Observe that $|\tau(\mathbf{v})-m k|$ and $\left|x_{\tau(\mathbf{v})}^{\mathbf{v}}-m k \xi\right|$ are (essentially) bounded by the total length of the bad segments and hence by $C \varepsilon m k$. Moreover, due to total ergodicity $\Lambda_{\ell}(g, \cdot)$ is concave on $\mathcal{U}_{0}$ and hence continuous in its interior. Thus, we can choose $\varepsilon>0$ small enough so that

$$
m k \Lambda_{\ell}(g, \zeta)+m k \delta>\tau(\mathbf{v}) \Lambda_{\ell}(g, \eta(\mathbf{v})) .
$$

(3.8) would then follow if we show

$$
\lim _{m \rightarrow \infty} \sum_{\mathbf{v}} \mathbb{P}\left\{\sum_{x_{0, m k} \in \Pi_{m k, m k \xi}^{\mathbf{v}}} e^{m k R_{m k}^{\ell}(g)} \geq e^{\tau(\mathbf{v})\left(\Lambda_{\ell}(g, \eta(\mathbf{v}))+\log |\mathcal{R}|\right)+3 m k \delta}\right\}=0 .
$$

This, in turn, follows from showing

$$
\begin{aligned}
\lim _{m \rightarrow \infty} \sum_{\mathbf{v}} \mathbb{P}\left\{\sum_{x_{0, m k} \in \Pi_{m k, m k \xi}^{\mathbf{v}}} e^{\tau(\mathbf{v}) R_{\tau(\mathbf{v})}^{\ell}(g)\left(\omega^{\mathbf{v}}, x_{0, \tau(\mathbf{v})+\ell}^{\mathbf{v}}\right)}\right. & \\
& \left.\geq e^{\tau(\mathbf{v})\left(\Lambda_{\ell}(g, \eta(\mathbf{v}))+\log |\mathcal{R}|\right)+2 m k \delta}\right\}=0 .
\end{aligned}
$$

To justify the step to (3.9), first delete all terms from

$$
m k R_{m k}^{\ell}(g)=\sum_{i=0}^{m k-1} g\left(T_{x_{i}} \omega, z_{i+1, i+\ell}\right)
$$

that depend on $\omega$ or $\left(z_{i}\right)$ outside of good slabs. Since $g \leq 0$ this goes in the right direction. The remaining terms can be written in the form $\sum_{i} g\left(T_{x_{i}^{\mathbf{v}}} \omega^{\mathbf{v}}, z_{i+1, i+\ell}^{\mathbf{v}}\right)$ for a certain subset of indices $i \in\{0, \ldots, \tau(\mathbf{v})-1\}$. Then add in the terms for the remaining indices to capture the entire sum

$$
\tau(\mathbf{v}) R_{\tau(\mathbf{v})}^{\ell}(g)\left(\omega^{\mathbf{v}}, x_{0, \tau(\mathbf{v})+\ell}^{\mathbf{v}}\right)=\sum_{i=0}^{\tau(\mathbf{v})-1} g\left(T_{x_{i}} \omega^{\mathbf{v}}, z_{i+1, i+\ell}^{\mathbf{v}}\right) .
$$

The terms added correspond to terms that originally straddled good and bad segments. Hence since $g$ is local in its dependence on both $\omega$ and $z_{1, \infty}$ there are at most $C \varepsilon m k$ such terms. Since $g$ is bounded, choosing $\varepsilon$ small enough allows us to absorb all such terms into one $m k \delta$ error.

Observing that $\omega^{\mathbf{v}}$ has the same distribution as $\omega$, adding more paths in the sum inside the probability, and recalling that $|\tau(\mathbf{v})-m k| \leq C m k \varepsilon$, we see that it is enough to prove

$$
\lim _{m \rightarrow \infty} \sum_{\mathbf{v}} \mathbb{P}\left\{\sum_{x_{0, \tau(\mathbf{v})} \in \Pi_{\tau(\mathbf{v}), \tau(\mathbf{v}) \eta(\mathbf{v})}} e^{\tau(\mathbf{v}) R_{\tau(\mathbf{v})}^{\ell}(g)} \geq e^{\tau(\mathbf{v})\left(\Lambda_{\ell}(g, \eta(\mathbf{v}))+\log |\mathcal{R}|\right)+\tau(\mathbf{v}) \delta}\right\}=0 .
$$

By Lemma 3.3, concentration inequality Lemma B.1, and $\tau(\mathbf{v}) \geq m k / 2$, the sum of probabilities above is bounded by $C(m k)^{c_{1}} e^{m k h(\varepsilon)-B \delta^{2} m k / 2} \leq$ $C(m k)^{c_{1}} e^{-\left(\delta_{1}-h(\varepsilon)\right) k m}$ for another small positive constant $\delta_{1}$. Choosing $\varepsilon$ small enough shows convergence to 0 exponentially fast in $m$.

We have verified the original goal (3.6) and thereby completed the proof of Theorem 2.6. 
4 Quenched large deviations and entropy representation of the free energy

Standing assumptions for this section are $\mathcal{R} \subset \mathbb{Z}^{d}$ is finite and $\left(\Omega, \mathfrak{S}, \mathbb{P},\left\{T_{z}\right.\right.$ : $z \in \mathcal{G}\}$ ) is a measurable ergodic dynamical system. We begin with those results that do not need further assumptions on $\mathbb{P}$, first the quenched large deviation principle for the walk under the polymer measures. The theorem below assumes $\Lambda_{\ell}(g)$ finite; recall Remark 2.3 for conditions that guarantee this.

We employ the following notation for lower semicontinuous regularization of a function of several variables:

$$
F^{\operatorname{lsc}(x)}(x, y)=\lim _{r \searrow 0} \inf _{z:|z-x|<r} F(z, y),
$$

and analogously for upper semicontinuous regularization.

Theorem 4.1 Let $\ell \geq 0$ and let $g: \Omega \times \mathcal{R}^{\ell} \rightarrow \mathbb{R}$. Assume $g \in \mathcal{L}$ and that $\Lambda_{\ell}(g)$ is finite. Then for $\mathbb{P}$-a.e. $\omega$, the distributions $Q_{n}^{g, \omega}\left\{X_{n} / n \in \cdot\right\}$ on $\mathbb{R}^{d}$ satisfy an LDP with rate function

$$
I^{g}(\zeta)=\Lambda_{\ell}(g)-\Lambda_{\ell}^{\mathrm{usc}(\zeta)}(g, \zeta) .
$$

This means that the following bounds hold:

$$
\begin{aligned}
\varlimsup_{n \rightarrow \infty} n^{-1} \log Q_{n}^{g, \omega}\left\{X_{n} / n \in A\right\} & \leq-\inf _{\zeta \in A} I^{g}(\zeta) \text { for closed } A \subset \mathbb{R}^{d} \\
\text { and } \varliminf_{n \rightarrow \infty} n^{-1} \log Q_{n}^{g, \omega}\left\{X_{n} / n \in O\right\} & \geq-\inf _{\zeta \in O} I^{g}(\zeta) \text { for open } O \subset \mathbb{R}^{d} .
\end{aligned}
$$

Rate function $I^{g}: \mathbb{R}^{d} \rightarrow[0, \infty]$ is convex, and on $\mathcal{U}$ also finite and continuous.

Proof of Theorem 4.1 Let $O \subset \mathbb{R}^{d}$ be open, and $\zeta \in \mathcal{U} \cap O$. Then $\hat{x}_{n}(\zeta) \in n O$ for large $n$.

$$
\begin{aligned}
& \varliminf_{n \rightarrow \infty} n^{-1} \log Q_{n}^{g, \omega}\left\{X_{n} / n \in O\right\} \\
& \geq \underset{n \rightarrow \infty}{\lim _{n \rightarrow \infty}}\left\{n^{-1} \log E\left[e^{n R_{n}^{\ell}(g)} \mathbb{1}\left\{X_{n}=\hat{x}_{n}(\zeta)\right\}\right]-n^{-1} \log E\left[e^{n R_{n}^{\ell}(g)}\right]\right\} \\
& =\Lambda_{\ell}(g, \zeta)-\Lambda_{\ell}(g) .
\end{aligned}
$$

A supremum over an open set does not feel the difference between a function and its upper semicontinuous regularization, and so we get the lower large deviation bound:

$$
\varliminf_{n \rightarrow \infty} n^{-1} \log Q_{n}^{g, \omega}\left\{X_{n} / n \in O\right\} \geq-\inf _{\zeta \in O}\left\{\Lambda_{\ell}(g)-\Lambda_{\ell}^{\mathrm{usc}}(g, \zeta)\right\} .
$$

For a closed set $K \subset \mathbb{R}^{d}$ and $\delta>0$ Lemma 2.8 implies

$$
\begin{aligned}
\varlimsup_{n \rightarrow \infty} n^{-1} \log Q_{n}^{g, \omega}\left\{X_{n} / n \in K\right\} & \leq-\lim _{\delta \searrow 0} \inf _{\zeta \in K_{\delta}}\left\{\Lambda_{\ell}(g)-\Lambda_{\ell}(g, \zeta)\right\} \\
& \leq-\lim _{\delta \searrow 0} \inf _{\zeta \in K_{\delta}}\left\{\Lambda_{\ell}(g)-\Lambda_{\ell}^{\text {usc }}(g, \zeta)\right\} \\
& =-\inf _{\zeta \in K}\left\{\Lambda_{\ell}(g)-\Lambda_{\ell}^{\text {usc }}(g, \zeta)\right\} .
\end{aligned}
$$


The last limit $\delta \searrow 0$ follows from the compactness of $\mathcal{U}$. Properties of $I^{g}$ follow from Theorem 2.5(b) and (f).

Remark 4.2 Since the rate function $I^{g}$ is convex, it is the convex dual of the limiting logarithmic moment generating function

$$
\sigma(t)=\lim _{n \rightarrow \infty} n^{-1} \log E^{Q_{n}^{g, \omega}}\left(e^{t \cdot X_{n}}\right)=\Lambda_{\ell}\left(g+t \cdot z_{1}\right)-\Lambda_{\ell}(g)
$$

on $\mathbb{R}^{d}$. This gives the identity

$$
-\Lambda_{\ell}^{\mathrm{usc}}(g, \zeta)=\sup _{t \in \mathbb{R}^{d}}\left\{\zeta \cdot t-\Lambda_{\ell}\left(g+t \cdot z_{1}\right)\right\} .
$$

As a corollary of Theorem 4.1, we state a level 1 LDP for RWRE (see Example 1.4).

Corollary 4.3 Let $d \geq 1$. Consider $R W R E$ on $\mathbb{Z}^{d}$ in an ergodic environment with a finite set $\mathcal{R} \subset \mathbb{Z}^{d}$ of admissible steps. Assume $g(\omega, z)=\log p_{z}(\omega)$ is a member of $\mathcal{L}$. Then there exists a continuous, convex rate function $I: \mathcal{U} \rightarrow$ $[0, \infty)$ such that, for $\mathbb{P}$-a.e. $\omega$, the distributions $Q^{\omega}\left\{X_{n} / n \in \cdot\right\}$ on $\mathcal{U}$ satisfy an $L D P$ with rate $I$. For $\zeta \in \operatorname{ri} \mathcal{U}, I(\zeta)$ is the limit of point probabilities:

$$
I(\zeta)=-\lim _{n \rightarrow \infty} n^{-1} \log Q_{0}^{\omega}\left\{X_{n}=\hat{x}_{n}(\zeta)\right\} \quad \text { a.s. }
$$

With either a compact $\Omega$ or an i.i.d. directed setting, the LDP of Theorem 4.1 can be obtained by contraction from the higher level LDPs of [26]. This is the route to linking $\Lambda_{\ell}(g, \zeta)$ with entropy. First we define the entropy.

The joint evolution of the environment and the walk give a Markov chain $\left(T_{X_{n}} \omega, Z_{n+1, n+\ell}\right)$ on the state space $\boldsymbol{\Omega}_{\ell}=\Omega \times \mathcal{R}^{\ell}$. Elements of $\boldsymbol{\Omega}_{\ell}$ are denoted by $\eta=\left(\omega, z_{1, \ell}\right)$. The transition kernel is

$$
\hat{p}_{\ell}\left(\eta, S_{z}^{+} \eta\right)=\frac{1}{|\mathcal{R}|} \text { for } z \in \mathcal{R} \text { and } \eta=\left(\omega, z_{1, \ell}\right) \in \boldsymbol{\Omega}_{\ell}
$$

where the transformations $S_{z}^{+}$are defined by $S_{z}^{+}\left(\omega, z_{1, \ell}\right)=\left(T_{z_{1}} \omega,\left(z_{2, \ell}, z\right)\right)$. An entropy $H_{\ell}$ that is naturally associated to this Markov chain and reflects the role of the background measure is defined as follows. Let $\mu_{0}$ denote the $\Omega$-marginal of a probability measure $\mu \in \mathcal{M}_{1}\left(\boldsymbol{\Omega}_{\ell}\right)$. Define

$$
H_{\ell}(\mu)= \begin{cases}\inf \left\{H\left(\mu \times q \mid \mu \times \hat{p}_{\ell}\right): q \in \mathcal{Q}\left(\boldsymbol{\Omega}_{\ell}\right) \text { with } \mu q=\mu\right\} & \text { if } \mu_{0} \ll \mathbb{P} \\ \infty & \text { otherwise }\end{cases}
$$

The infimum is over Markov kernels $q$ on $\boldsymbol{\Omega}_{\ell}$ that fix $\mu$. Inside the braces the familiar relative entropy is

$$
H\left(\mu \times q \mid \mu \times \hat{p}_{\ell}\right)=\int_{\boldsymbol{\Omega}_{\ell}} \sum_{z \in \mathcal{R}} q\left(\eta, S_{z}^{+} \eta\right) \log \frac{q\left(\eta, S_{z}^{+} \eta\right)}{\hat{p}_{\ell}\left(\eta, S_{z}^{+} \eta\right)} \mu(d \eta) .
$$


Obviously $q\left(\eta, S_{z}^{+} \eta\right)$ is not the most general Markov kernel on $\boldsymbol{\Omega}_{\ell}$. But the entropy cannot be finite unless the kernel is supported on shifts $S_{z}^{+} \eta$, so we might as well restrict to this case. $H_{\ell}: \mathcal{M}_{1}\left(\boldsymbol{\Omega}_{\ell}\right) \rightarrow[0, \infty]$ is convex. (The argument for this can be found at the end of Section 4 in [25].)

The quenched free energy has this variational characterization for $g \in \mathcal{L}$ (Theorem 2.3 in [26]):

$$
\Lambda_{\ell}(g)=\sup _{\mu \in \mathcal{M}_{1}\left(\boldsymbol{\Omega}_{\ell}\right), c>0}\left\{E^{\mu}[\min (g, c)]-H_{\ell}(\mu)\right\} .
$$

Our goal is to find such characterizations for the point-to-point free energy.

Theorem 4.4 Fix $\ell \geq 1$ and let $g: \boldsymbol{\Omega}_{\ell} \rightarrow \mathbb{R}$. Assume $g \in \mathcal{L}$ and that $\Lambda_{\ell}(g)$ is finite. Assume that $\Omega$ is a compact metric space. Then for $\zeta \in \operatorname{ri} \mathcal{U}$

$$
\Lambda_{\ell}(g, \zeta)=\sup _{\substack{\mu \in \mathcal{M}_{1}\left(\boldsymbol{\Omega}_{\ell}\right): E^{\mu}\left[Z_{1}\right]=\zeta \\ c>0}}\left\{E^{\mu}[\min (g, c)]-H_{\ell}(\mu)\right\} .
$$

For $\zeta \in \mathcal{U} \backslash$ ri $\mathcal{U},(4.9)$ is valid under the following assumption: $\mathbb{P}$ is ergodic under the smaller set of shifts $\left\{T_{z}: z \in \mathcal{R}_{0}\right\}$, where $\mathcal{R}_{0}=\mathcal{U}_{0} \cap \mathcal{R}$ and $\mathcal{U}_{0}$ is the unique face of $\mathcal{U}$ such that $\zeta \in$ ri $\mathcal{U}_{0}$.

Proof With $\Omega$ assumed compact, Theorem 3.1 of [26] gives a quenched LDP for the distributions $Q_{n}^{g, \omega}\left\{R_{n}^{\ell} \in \cdot\right\}$ of the empirical measure $R_{n}^{\ell}$ defined by (2.2), with rate function

$$
I_{2}^{g}(\mu)=\left(\inf _{c>0}\left\{H_{\ell}(\mu)-E^{\mu}[g \wedge c]\right\}\right)^{\operatorname{lsc}(\mu)}+\Lambda_{\ell}(g) .
$$

The contraction principle of large deviation theory [9, Theorem 4.2.1] then recovers the LDP in Theorem 4.1 and gives this representation for the rate function $I^{g}$ :

$$
\begin{aligned}
I^{g}(\zeta) & =\inf _{\mu \in \mathcal{M}_{1}\left(\boldsymbol{\Omega}_{\ell}\right): E^{\mu}\left[Z_{1}\right]=\zeta}\left(\inf _{c>0}\left\{H_{\ell}(\mu)-E^{\mu}[g \wedge c]\right\}\right)^{\operatorname{lsc}(\mu)}+\Lambda_{\ell}(g) \\
& =\left(\inf _{\substack{\mu \mathcal{M}_{1}\left(\boldsymbol{\Omega}_{\ell}\right): E^{\mu}\left[Z_{1}\right]=\zeta \\
c>0}}\left\{H_{\ell}(\mu)-E^{\mu}[g \wedge c]\right\}\right)^{\operatorname{lsc}(\zeta)}+\Lambda_{\ell}(g) n \\
& =-\left(\sup _{\substack{\mu \in \mathcal{M}_{1}\left(\boldsymbol{\Omega}_{\ell}\right): E^{\mu}\left[Z_{1}\right]=\zeta \\
c>0}}\left\{E^{\mu}[g \wedge c]-H_{\ell}(\mu)\right\}\right)^{\mathrm{usc}(\zeta)}+\Lambda_{\ell}(g) .
\end{aligned}
$$

In the second equality above, compact sublevel sets of the higher level rate function (simply a consequence of assuming $\Omega$ compact) and the continuity of $\mu \mapsto E^{\mu}\left[Z_{1}\right]$ allow us to take the lower semicontinuous regularization outside the contraction.

Comparing (4.1) with the above gives

$$
\Lambda_{\ell}^{\mathrm{usc}(\zeta)}(g, \zeta)=\left(\sup _{\substack{\mu \in \mathcal{M}_{1}\left(\boldsymbol{\Omega}_{\ell}\right): E^{\mu}\left[Z_{1}\right]=\zeta \\ c>0}}\left\{E^{\mu}[\min (g, c)]-H_{\ell}(\mu)\right\}\right)^{\mathrm{usc}(\zeta)}
$$


For $\zeta \in$ ri $\mathcal{U}$ the upper semicontinuous regularizations can be dropped. On the left we have continuity on ri $\mathcal{U}$ by the concavity from Theorem $2.5(\mathrm{~b})$. On the right one can check by hand that $\sup _{c>0}\left\{E^{\mu}[g \wedge c]-H_{\ell}(\mu)\right\}$ is concave in $\mu$, and this concavity extends to the function of $\zeta$ defined by the supremum over $\mu$. The function inside the parentheses is bounded above by (4.11) because $I^{g}(\zeta) \geq 0$. It is bounded below because we can take any probability measure $\alpha$ on $\mathcal{R}$ with expectation $\zeta$, and then $\mu=\mathbb{P} \otimes \alpha^{\otimes \ell}$ is an admissible argument in the supremum. Thus on the right too we have a finite concave function of $\zeta$ and hence continuous on the relative interior.

Next we extend the result to the relative boundary of $\mathcal{U}$, with the additional assumption of ergodicity of $\mathbb{P}$.

Suppose first that $\zeta$ is an extreme point of $\mathcal{U}$. Then $\zeta=\tilde{z} \in \mathcal{R}, \mathcal{R}_{0}=\{\tilde{z}\}$, and the unique path to $\hat{x}_{n}(\tilde{z})=n \tilde{z}$ uses only $\tilde{z}$-steps. (4.9) is proved by comparing the left and right sides. By ergodicity, $\Lambda_{\ell}(g, \tilde{z})=\mathbb{E} g(\omega,(\tilde{z}, \ldots, \tilde{z}))+$ $\log \hat{p}_{\tilde{z}}$. On the right $E^{\mu}\left[Z_{1}\right]=\tilde{z}$ forces $\mu$ to be supported on $\Omega \times\{(\tilde{z}, \ldots, \tilde{z})\}$. A kernel that fixes $\mu$ can only use the shift $T_{\tilde{z}}$, and $\mu_{0}$ must be $T_{\tilde{z}}$-invariant. By ergodicity of $\mathbb{P}$ under $T_{\tilde{z}}, T_{\tilde{z}}$-invariance and $\mu_{0} \ll \mathbb{P}$ imply that $\mu_{0}=\mathbb{P}$. Thus on the right in (4.9) the only measure is $\mu=\mathbb{P} \otimes \delta_{(\tilde{z}, \ldots, \tilde{z})}$, and the equality holds.

In the remaining case $\mathcal{U}_{0}$ is the unique face such that $\zeta \in$ ri $\mathcal{U}_{0}$. Then $\mathcal{U}_{0}=$ co $\mathcal{R}_{0}$ where $\mathcal{R}_{0}=\mathcal{U}_{0} \cap \mathcal{R}$, and any path to $\hat{x}_{n}(\zeta)$ will use only $\mathcal{R}_{0}$-steps. This case reduces to the one already proved, because all the quantities in (4.9) are the same as those in a new model where $\mathcal{R}$ is replaced by $\mathcal{R}_{0}$ and then $\mathcal{U}$ is replaced by $\mathcal{U}_{0}$. (Except for the extra terms coming from renormalizing the restricted jump kernel $\left\{\hat{p}_{z}\right\}_{z \in \mathcal{R}_{0}}$.) For example, $E^{\mu}\left[Z_{1}\right]=\zeta$ forces $\mu$ to be supported on $\Omega \times \mathcal{R}_{0}^{\ell}$, and consequently any kernel $q\left(\eta, S_{z}^{+} \eta\right)$ that fixes $\mu$ is supported on shifts by $z \in \mathcal{R}_{0}$.

The assumption $\ell \geq 1$ in Theorem 4.4 guarantees that the expectation $E^{\mu}\left[Z_{1}\right]$ makes sense for $\mu \in \mathcal{M}_{1}\left(\boldsymbol{\Omega}_{\ell}\right)$. Since $g$ can be composed with a projection this assumption is not a restriction on $g$.

Remark 4.5 We emphasize that the restriction to compact $\Omega$ in Theorem 4.4 is entirely due to the fact that Theorem 3.1 of [26] gives only a weak quenched LDP (weak in the sense that the upper bound is valid only for compact sets). Thus to obtain the rate function $I^{g}$ of (4.1) as a contraction we assume $\Omega$ compact. This is unsatisfactory because for the simplest directed polymer with unbounded potential we would like to use a non-compact $\Omega$ such as $\mathbb{R}^{\mathbb{Z}^{d}}$. Fortunately in the most important case, namely i.i.d. directed, the LDP of [26] is a full LDP, and we can drop the compactness assumption. We turn to this case.

Let $\Omega=\Gamma^{\mathbb{Z}^{d}}$ be a product space with shifts $\left\{T_{x}\right\}$ and $\mathbb{P}$ an i.i.d. product measure as in Example 1.1. Assume $0 \notin \mathcal{U}$. Then the free energies $\Lambda_{\ell}(g)$ and $\Lambda_{\ell}(g, \zeta)$ are deterministic (that is, the $\mathbb{P}$-a.s. limits are independent of the environment $\omega)$ and $\Lambda_{\ell}(g, \zeta)$ is a continuous, concave function of $\zeta \in \mathcal{U}$. 
Assume also that $\Gamma$ is a separable metric space, and that $\mathfrak{S}$ is the product of Borel $\sigma$-algebras, thereby also the Borel $\sigma$-algebra of $\Omega$.

To utilize convex analysis we put the space $\mathcal{M}$ of finite Borel measures on $\boldsymbol{\Omega}_{\ell}$ in duality with $C_{b}\left(\boldsymbol{\Omega}_{\ell}\right)$, the space of bounded continuous functions on $\boldsymbol{\Omega}_{\ell}$, via integration: $\langle f, \mu\rangle=\int f d \mu$. Give $\mathcal{M}$ the weak topology generated by $C_{b}\left(\boldsymbol{\Omega}_{\ell}\right)$. Metrize $C_{b}\left(\boldsymbol{\Omega}_{\ell}\right)$ with the supremum norm. The limit definition (2.3) shows that $\Lambda_{\ell}(g)$ and $\Lambda_{\ell}(g, \zeta)$ are Lipschitz in $g$, uniformly in $\zeta . H_{\ell}$ is extended to $\mathcal{M}$ by setting $H_{\ell}(\mu)=\infty$ for measures $\mu$ that are not probability measures.

For $g \in C_{b}\left(\boldsymbol{\Omega}_{\ell}\right)$ (4.8) says that $\Lambda_{\ell}(g)=H_{\ell}^{*}(g)$, the convex conjugate of $H_{\ell}$. The double convex conjugate

$$
H_{\ell}^{* *}(\mu)=\Lambda_{\ell}^{*}(\mu)=\sup _{f \in C_{b}\left(\boldsymbol{\Omega}_{\ell}\right)}\left\{E^{\mu}[f]-\Lambda_{\ell}(f)\right\}, \quad \mu \in \mathcal{M}_{1}\left(\boldsymbol{\Omega}_{\ell}\right)
$$

is equal to the lower semicontinuous regularization $H_{\ell}^{\mathrm{lsc}}$ of $H_{\ell}$ (Propositions 3.3 and 4.1 in [11] or Theorem 5.18 in [24]). Since relative entropy is lower semicontinuous, (4.6) implies that

$$
H_{\ell}^{* *}(\mu)=H_{\ell}(\mu) \quad \text { for } \mu \in \mathcal{M}_{1}\left(\boldsymbol{\Omega}_{\ell}\right) \text { such that } \mu_{0} \ll \mathbb{P} \text {. }
$$

There is a quenched LDP for the distributions $Q_{n}^{g, \omega}\left\{R_{n}^{\ell} \in \cdot\right\}$, where $R_{n}^{\ell}$ is the emprirical measure defined in (2.2). The rate function of this LDP is $H_{\ell}^{* *}$ (Theorems 3.1 and 3.3 of [26]).

The reader may be concerned about considering the $\mathbb{P}$-a.s. defined functionals $\Lambda_{\ell}(g)$ or $\Lambda_{\ell}(g, \zeta)$ on the possibly non-separable function space $C_{b}\left(\boldsymbol{\Omega}_{\ell}\right)$. However, for bounded functions we can integrate over the limits (2.3) and (2.4) and define the free energies without any "a.s. ambiguity", so for example

$$
\Lambda_{\ell}(g, \zeta)=\lim _{n \rightarrow \infty} n^{-1} \mathbb{E}\left(\log E\left[e^{n R_{n}^{\ell}(g)} \mathbb{1}\left\{X_{n}=\hat{x}_{n}(\zeta)\right\}\right]\right)
$$

We extend the duality set-up to involve point to point free energy.

Theorem 4.6 Let $\Omega=\Gamma^{\mathbb{Z}^{d}}$ be a product of separable metric spaces with Borel $\sigma$-algebra $\mathfrak{S}$, shifts $\left\{T_{x}\right\}$, and an an i.i.d. product measure $\mathbb{P}$. Assume $0 \notin \mathcal{U}$. With $\ell \geq 1$, let $\mu \in \mathcal{M}_{1}\left(\boldsymbol{\Omega}_{\ell}\right)$ and $\zeta=E^{\mu}\left[Z_{1}\right]$. Then

$$
H_{\ell}^{* *}(\mu)=\sup _{g \in C_{b}\left(\boldsymbol{\Omega}_{\ell}\right)}\left\{E^{\mu}[g]-\Lambda_{\ell}(g, \zeta)\right\}
$$

On the other hand, for $f \in C_{b}\left(\boldsymbol{\Omega}_{\ell}\right)$ and $\zeta \in \mathcal{U}$,

$$
\Lambda_{\ell}(f, \zeta)=\sup _{\mu \in \mathcal{M}_{1}\left(\boldsymbol{\Omega}_{\ell}\right): E^{\mu}\left[Z_{1}\right]=\zeta}\left\{E^{\mu}[f]-H_{\ell}^{* *}(\mu)\right\} .
$$

Equation (4.16) is valid also when $H_{\ell}^{* *}(\mu)$ is replaced with $H_{\ell}(\mu)$. 
Proof With fixed $\zeta$, introduce the convex conjugate of $\Lambda_{\ell}(g, \zeta)$ by

$$
\Lambda_{\ell}^{*}(\mu, \zeta)=\sup _{g \in C_{b}\left(\boldsymbol{\Omega}_{\ell}\right)}\left\{E^{\mu}[g]-\Lambda_{\ell}(g, \zeta)\right\} .
$$

Taking $g\left(\omega, z_{1, \ell}\right)=a \cdot z_{1}$ gives $\Lambda_{\ell}^{*}(\mu, \zeta) \geq a \cdot\left(E^{\mu}\left[Z_{1}\right]-\zeta\right)-\log \left|\mathcal{R}_{0}\right|$. Thus $\Lambda_{\ell}^{*}(\mu, \zeta)=\infty$ unless $E^{\mu}\left[Z_{1}\right]=\zeta$.

From Theorems 2.5 and 2.6, $E^{\mu}[g]-\Lambda_{\ell}(g, \zeta)$ is concave in $g$, convex in $\zeta$, and continuous in both over $C_{b}\left(\boldsymbol{\Omega}_{\ell}\right) \times \mathcal{U}$. Since $\mathcal{U}$ is compact we can apply a minimax theorem such as König's theorem [16,24]. Utilizing (2.5),

$$
\begin{aligned}
\Lambda_{\ell}^{*}(\mu) & =\sup _{g \in C_{b}\left(\boldsymbol{\Omega}_{\ell}\right)}\left\{E^{\mu}[g]-\Lambda_{\ell}(g)\right\} \\
& =\sup _{g \in C_{b}\left(\boldsymbol{\Omega}_{\ell}\right)} \inf _{\zeta \in \mathcal{U}}\left\{E^{\mu}[g]-\Lambda_{\ell}(g, \zeta)\right\}=\inf _{\zeta \in \mathcal{U}} \Lambda_{\ell}^{*}(\mu, \zeta) .
\end{aligned}
$$

Thus, if $E^{\mu}\left[Z_{1}\right]=\zeta$, then $\Lambda_{\ell}^{*}(\mu)=\Lambda_{\ell}^{*}(\mu, \zeta)$. Since $H_{\ell}^{* *}(\mu)=\Lambda_{\ell}^{*}(\mu),(4.15)$ follows from (4.17).

By double convex duality (Fenchel-Moreau theorem, see e.g. [24]), for $f \in$ $C_{b}\left(\boldsymbol{\Omega}_{\ell}\right)$,

$$
\Lambda_{\ell}(f, \zeta)=\sup _{\mu}\left\{E^{\mu}[f]-\Lambda_{\ell}^{*}(\mu, \zeta)\right\}=\sup _{\mu: E^{\mu}\left[Z_{1}\right]=\zeta}\left\{E^{\mu}[f]-\Lambda_{\ell}^{*}(\mu)\right\}
$$

and (4.16) follows.

To replace $H_{\ell}^{* *}(\mu)$ with $H_{\ell}(\mu)$ in (4.16), we first consider the case $\zeta \in \operatorname{ri} \mathcal{U}$ and reason as was done after (4.12):

$$
\begin{aligned}
\sup _{\mu \in \mathcal{M}_{1}\left(\boldsymbol{\Omega}_{\ell}\right): E^{\mu}\left[Z_{1}\right]=\zeta}\left\{E^{\mu}[f]-H_{\ell}^{* *}(\mu)\right\} \\
=\sup _{\mu \in \mathcal{M}_{1}\left(\boldsymbol{\Omega}_{\ell}\right): E^{\mu}\left[Z_{1}\right]=\zeta}\left\{E^{\mu}[f]-H_{\ell}(\mu)\right\}^{\operatorname{usc}(\mu)} \\
=\left(\sup _{\mu \in \mathcal{M}_{1}\left(\boldsymbol{\Omega}_{\ell}\right): E^{\mu}\left[Z_{1}\right]=\zeta}\left\{E^{\mu}[f]-H_{\ell}(\mu)\right\}\right)^{\operatorname{usc}(\zeta)} \\
=\sup _{\mu \in \mathcal{M}_{1}\left(\boldsymbol{\Omega}_{\ell}\right): E^{\mu}\left[Z_{1}\right]=\zeta}\left\{E^{\mu}[f]-H_{\ell}(\mu)\right\} .
\end{aligned}
$$

The first equality is the continuity of $\mu \mapsto E^{\mu}[f]$. The second is a consequence of the compact sublevel sets of $\left\{\mu: H_{\ell}^{* *}(\mu) \leq c\right\}$. This compactness follows from the exponential tightness in the LDP controlled by the rate $H_{\ell}^{* *}$, given by Theorem 3.3 in [26]. The last equality follows because concavity gives continuity on ri $\mathcal{U}$.

Then, as in the proof of the last part of Theorem 4.4, we can use the just proved identity (namely, (4.16) with $H_{\ell}^{* *}(\mu)$ replaced by $H_{\ell}(\mu)$ ) in a new setting where the set of admissible steps is $\mathcal{R}_{0}=\mathcal{U}_{0} \cap \mathcal{R}$ where $\mathcal{U}_{0}$ is the unique face that contains $\zeta$ in its relative interior. This way we get all $\zeta \in \mathcal{U}$.

Next we extend the duality to certain $L^{p}$ functions. 
Corollary 4.7 Same assumptions on $\Omega, \mathbb{P}$ and $\mathcal{R}$ as in Theorem 4.6. Let $\mu \in \mathcal{M}_{1}\left(\boldsymbol{\Omega}_{\ell}\right)$ and $\zeta=E^{\mu}\left[Z_{1}\right]$. Then the inequalities

$$
E^{\mu}[g]-\Lambda_{\ell}(g) \leq H_{\ell}^{* *}(\mu)
$$

and

$$
E^{\mu}[g]-\Lambda_{\ell}(g, \zeta) \leq H_{\ell}^{* *}(\mu)
$$

are valid for all functions $g$ such that $g\left(\cdot, z_{1, \ell}\right)$ is local and in $L^{p}(\mathbb{P})$ for all $z_{1, \ell}$ and some $p>d$, and $g$ is either bounded above or bounded below.

Proof Since $\Lambda_{\ell}(g, \zeta) \leq \Lambda_{\ell}(g)$, (4.18) is a consequence of (4.19). Let $\mathcal{H}$ denote the class of functions $g$ that satisfy (4.19). $\mathcal{H}$ contains bounded continuous local functions by (4.15).

Bounded pointwise convergence implies $L^{p}$ convergence. So by the $L^{p}$ continuity of $\Lambda_{\ell}(g, \zeta)$ (Lemma 3.1(b)), $\mathcal{H}$ is closed under bounded pointwise convergence of local functions with common support. General principles now imply that $\mathcal{H}$ contains all bounded local Borel functions. To reach the last generalization to functions bounded from only one side, observe that their truncations converge both monotonically and in $L^{p}$, thereby making both $E^{\mu}[g]$ and $\Lambda_{\ell}(g, \zeta)$ converge.

Equation (4.16) gives us a variational representation for $\Lambda_{\ell}(g, \zeta)$ but only for bounded continuous $g$. We come finally to one of our main results, the variational representation for general potentials $g$.

Theorem 4.8 Let $\Omega=\Gamma^{\mathbb{Z}^{d}}$ be a product of separable metric spaces with Borel $\sigma$-algebra $\mathfrak{S}$, shifts $\left\{T_{x}\right\}$, and an i.i.d. product measure $\mathbb{P}$. Assume $0 \notin \mathcal{U}$. Let $g: \boldsymbol{\Omega}_{\ell} \rightarrow \mathbb{R}$ be a function such that for each $z_{1, \ell} \in \mathcal{R}^{\ell}, g\left(\cdot, z_{1, \ell}\right)$ is a local function of $\omega$ and a member of $L^{p}(\mathbb{P})$ for some $p>d$. Then for all $\zeta \in \mathcal{U}$,

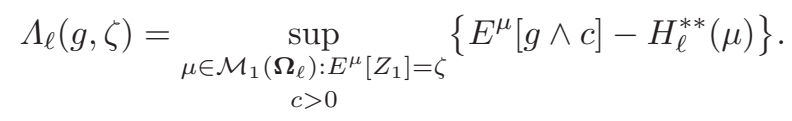

Equation (4.20) is valid also when $H_{\ell}^{* *}(\mu)$ is replaced with $H_{\ell}(\mu)$.

Proof From (4.19),

$$
\Lambda_{\ell}(g, \zeta) \geq \Lambda_{\ell}(g \wedge c, \zeta) \geq E^{\mu}[g \wedge c]-H_{\ell}^{* *}(\mu) .
$$

Supremum on the right over $c$ and $\mu$ gives

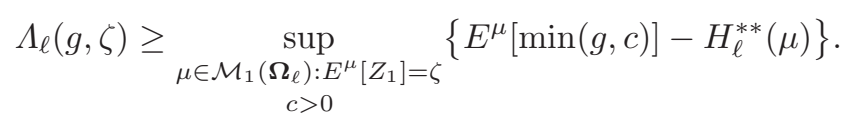

For the other direction, let $c<\infty$ and abbreviate $g^{c}=g \wedge c$. Let $g_{m} \in$ $C_{b}\left(\boldsymbol{\Omega}_{\ell}\right)$ be a sequence converging to $g^{c}$ in $L^{p}(\mathbb{P})$. Let $\varepsilon>0$. By (4.16) we can find $\mu_{m}$ such that $E^{\mu_{m}}\left[Z_{1}\right]=\zeta, H_{\ell}^{* *}\left(\mu_{m}\right)<\infty$ and

$$
\Lambda_{\ell}\left(g_{m}, \zeta\right) \leq \varepsilon+E^{\mu_{m}}\left[g_{m}\right]-H_{\ell}^{* *}\left(\mu_{m}\right) .
$$


Take $\beta>0$ and write

$$
\begin{aligned}
& \Lambda_{\ell}\left(g_{m}, \zeta\right) \leq \varepsilon+E^{\mu_{m}}\left[g^{c}\right]-H_{\ell}^{* *}\left(\mu_{m}\right)+\beta^{-1} E^{\mu_{m}}\left[\beta\left(g_{m}-g^{c}\right)\right] \\
& \leq \varepsilon+\sup \left\{E^{\mu}\left[g^{c}\right]-H_{\ell}^{* *}(\mu): c>0, E^{\mu}\left[Z_{1}\right]=\zeta\right\} \\
& \quad+\beta^{-1} \Lambda_{\ell}\left(\beta\left(g_{m}-g^{c}\right)\right)+\beta^{-1} H_{\ell}^{* *}\left(\mu_{m}\right) \\
& \leq \varepsilon+[\text { right-hand side of }(4.20)] \\
& +\varlimsup_{n \rightarrow \infty} \max _{x_{k}-x_{k-1} \in \mathcal{R}} n^{-1} \sum_{k=0}^{n-1}\left|g_{m}\left(T_{x_{k}} \omega, z_{1, \ell}\right)-g^{c}\left(T_{x_{k} \omega}, z_{1, \ell}\right)\right|+\beta^{-1} H_{\ell}^{* *}\left(\mu_{m}\right) \\
& \leq \varepsilon+[\operatorname{right-hand~side~of~}(4.20)] \\
& \quad+C \mathbb{E}\left[\max _{z_{1, \ell} \in \mathcal{R}^{\ell}}\left|g_{m}-g^{c}\right|^{p}\right]+\beta^{-1} H_{\ell}^{* *}\left(\mu_{m}\right) .
\end{aligned}
$$

The second inequality above used (4.18), and the last inequality used (3.1) and Chebyshev's inequality. Take first $\beta \rightarrow \infty$, then $m \rightarrow \infty$, and last $c \nearrow \varnothing$ and $\varepsilon \searrow 0$. Combined with (4.21), we have arrived at (4.20).

Dropping ${ }^{* *}$ requires no extra work. Since $H_{\ell} \geq H_{\ell}^{* *},(4.21)$ comes for free. For the complementary inequality simply replace $H_{\ell}^{* *}\left(\mu_{m}\right)$ with $H_{\ell}\left(\mu_{m}\right)$ in (4.22), as justified by the last line of Theorem 4.6.

\section{Directed polymer in the $L^{2}$ regime}

We illustrate the results of the previous section with an example. In a directed polymer with an i.i.d environment and high enough temperature, we use variational formula (4.3) to derive the value of $\Lambda_{1}(g, \zeta)$. Then we check that certain natural Markov chains are maximizers in the variational formula (4.20). When we use $H_{\ell}(\mu)$ rather than its l.s.c. regularization $H_{\ell}^{* *}(\mu)$, we can show uniqueness of the maximizer. The computations are done in a regime of weak disorder, under an $L^{2}$ assumption that is well-known in this context. We restrict to $\zeta \in \operatorname{ri} \mathcal{U}$, and the closer to the relative boundary we wish to take $\zeta$, the smaller we need to take the inverse temperature $\beta$.

The maximizing processes are basically the Markov chains constructed by Comets and Yoshida [4], though we admit a more general potential that can depend on the local environment and a step of the walk. A similar construction was also used by Yilmaz [34].

The setting is that of Example 1.2 with some further simplifications. $\Omega=$ $\mathbb{R}^{\mathbb{Z}^{d+1}}$ is a product space indexed by the space-time lattice where $d$ is the spatial dimension and the last coordinate direction is reserved for time. The environment is $\omega=\left(\omega_{x}\right)_{x \in \mathbb{Z}^{d+1}}$ and translations are $\left(T_{x} \omega\right)_{y}=\omega_{x+y}$. The coordinates $\omega_{x}$ are i.i.d. under $\mathbb{P}$. The set of admissible steps is of the form $\mathcal{R}=\left\{\left(z^{\prime}, 1\right): z^{\prime} \in \mathcal{R}^{\prime}\right\}$ for a finite set $\mathcal{R}^{\prime} \subset \mathbb{Z}^{d}$.

The situation will be in the weak disorder regime, so we have to assume that the difference of two $\mathcal{R}$-walks is at least 3 -dimensional. Precisely speaking, 
the additive subgroup of $\mathbb{Z}^{d+1}$ generated by $\mathcal{R}-\mathcal{R}=\{x-y: x, y \in \mathcal{R}\}$ is linearly isomorphic to some $\mathbb{Z}^{m}$, and we

assume that the dimension $m \geq 3$.

For example, $d \geq 3$ and $\mathcal{R}^{\prime}=\left\{ \pm e_{i}: 1 \leq i \leq d\right\}$ given by simple random walk qualifies.

The $P$-random walk has a kernel $\left(p_{z}\right)_{z \in \mathcal{R}}$. Earlier we assumed $p_{z}=|\mathcal{R}|^{-1}$, but this is not necessary for the results, any fixed kernel will do. We do assume $p_{z}>0$ for each $z \in \mathcal{R}$.

The potential is of the form $\beta g\left(\omega_{0}, z\right)$ where $\beta \in(0, \infty)$ is a parameter that tunes the strength of the interaction between the environment and the walk. We remove all finiteness concerns by assuming that

$$
\mathbb{E}\left[e^{c|g(\omega, z)|}\right]<\infty \quad \text { for some } c>0 \text { and all } z \in \mathcal{R} .
$$

Now $\Lambda_{\ell}(\beta g, \cdot)$ is well-defined and continuous on $\mathcal{U}$ for $\beta \in(0, c]$.

Define an averaged logarithmic moment generating function

$$
\lambda(\beta, \theta)=\log \sum_{z \in \mathcal{R}} p_{z} \mathbb{E}\left[e^{\beta g\left(\omega_{0}, z\right)+\theta \cdot z}\right] \quad \text { for } \beta \in[-c, c] \text { and } \theta \in \mathbb{R}^{d+1} .
$$

Under a fixed $\beta$, define the convex dual in the $\theta$-variable by

$$
\lambda^{*}(\beta, \zeta)=\sup _{\theta \in \mathbb{R}^{d+1}}\{\zeta \cdot \theta-\lambda(\beta, \theta)\}, \quad \zeta \in \mathcal{U} .
$$

For each $\beta \in[-c, c]$ and $\zeta \in$ ri $\mathcal{U}$ there exists $\theta \in \mathbb{R}^{d+1}$ such that $\nabla_{\theta} \lambda(\beta, \theta)=\zeta$ and this $\theta$ maximizes in (5.3). A point $\eta \in \mathbb{R}^{d+1}$ also maximizes if and only if

$$
(\theta-\eta) \cdot z \text { is constant over } z \in \mathcal{R} .
$$

Maximizers cannot be unique now because the last coordinate $\theta_{d+1}$ can vary freely without altering the expression in braces in (5.3). The spatial part $\theta^{\prime}=\left(\theta_{1}, \ldots, \theta_{d}\right)$ of a maximizer is unique if and only if $\mathcal{U}$ has nonempty $d$-dimensional interior.

Extend the random walk distribution $P$ to a two-sided walk $\left(X_{k}\right)_{k \in \mathbb{Z}}$ that satisfies $X_{0}=0$ and $Z_{i}=X_{i}-X_{i-1}$ for all $i \in \mathbb{Z}$, where the steps $\left(Z_{i}\right)_{i \in \mathbb{Z}}$ are i.i.d. $\left(p_{z}\right)$-distributed. For $n \in \mathbb{N}$ define forward and backward partition functions

$Z_{n}^{+}=E\left[e^{\beta \sum_{k=0}^{n-1} g\left(\omega_{X_{k}}, Z_{k+1}\right)+\theta \cdot X_{n}}\right]$ and $Z_{n}^{-}=E\left[e^{\beta \sum_{k=-n}^{-1} g\left(\omega_{X_{k}}, Z_{k+1}\right)-\theta \cdot X_{-n}}\right]$

and martingales

$$
W_{n}^{ \pm}=e^{-n \lambda(\beta, \theta)} Z_{n}^{ \pm} \quad \text { with } \quad \mathbb{E} W_{n}^{ \pm}=1 .
$$

The martingales $W_{n}^{ \pm}$are independent of each other. They are also functions of $(\beta, \theta)$ but there is no need to complicate the notation with this.

Suppose we have made assumptions that guarantee the $L^{1}$ convergence

$$
W_{n}^{ \pm} \rightarrow W_{\infty}^{ \pm}
$$


for some $(\beta, \theta)$ (a.s. convergence is automatic for nonnegative martingales). Then $\mathbb{E} W_{\infty}^{ \pm}=1$, and this combined with Kolmogorov's 0-1 law implies that $\mathbb{P}\left(W_{\infty}^{ \pm}>0\right)=1$. Then we can define a probability measure $\mu_{0}^{\theta}$ on $\Omega$ that is equivalent to $\mathbb{P}$ (in the sense that they share null sets) by

$$
\int_{\Omega} f(\omega) \mu_{0}^{\theta}(d \omega)=\mathbb{E}\left[W_{\infty}^{-} W_{\infty}^{+} f\right]
$$

Define a stochastic kernel from $\Omega$ to $\mathcal{R}$ by

$$
q_{0}^{\theta}(\omega, z)=p_{z} e^{\beta g\left(\omega_{0}, z\right)-\lambda(\beta, \theta)+\theta \cdot z} \frac{W_{\infty}^{+}\left(T_{z} \omega\right)}{W_{\infty}^{+}(\omega)} .
$$

Property $\sum_{z \in \mathcal{R}} q_{0}^{\theta}(\omega, z)=1$ comes from (one of) the identities

$$
W_{\infty}^{ \pm}=\sum_{z \in \mathcal{R}} p_{z} e^{\beta g\left(\omega_{a}( \pm), z\right)-\lambda(\beta, \theta)+\theta \cdot z} W_{\infty}^{ \pm} \circ T_{ \pm z} \quad \mathbb{P} \text {-a.s. }
$$

where $a^{(+)}=0$ and $a^{(-)}=-z$. These are inherited from the one-step Markov decomposition of $Z_{n}^{ \pm}$. For any $\ell \geq 0$, on $\boldsymbol{\Omega}_{\ell}$ we can define the probability measure $\mu^{\theta}$ by

$$
\mu^{\theta}\left(d \omega, z_{1, \ell}\right)=\mu_{0}^{\theta}(d \omega) q\left(\omega, z_{1}\right) q\left(T_{x_{1}} \omega, z_{2}\right) \cdots q\left(T_{x_{\ell-1}} \omega, z_{\ell}\right)
$$

where $x_{j}=z_{1}+\cdots+z_{j}$, and the stochastic kernel

$$
q^{\theta}\left(\left(\omega, z_{1, \ell}\right),\left(T_{z_{1}} \omega, z_{2, \ell} z\right)\right)=q_{0}^{\theta}\left(T_{x_{\ell}} \omega, z\right) .
$$

We think of $\beta$ fixed and $\theta$ varying and so include only $\theta$ in the notation of $\mu^{\theta}$ and $q^{\theta}$. Identities (5.6) can be used to show that $\mu^{\theta}$ is invariant under the kernel $q^{\theta}$, or explicitly, for any bounded measurable test function $f$,

$$
\sum_{z_{1, \ell}, z} \int_{\Omega} \mu^{\theta}\left(d \omega, z_{1, \ell}\right) q_{0}^{\theta}\left(T_{x_{\ell}} \omega, z\right) f\left(T_{z_{1}} \omega, z_{2, \ell} z\right)=\int_{\boldsymbol{\Omega}_{\ell}} f d \mu^{\theta} .
$$

By Lemma 4.1 of [25] the Markov chain with transition $q^{\theta}$ started with $\mu^{\theta}$ is an ergodic process. Let us call in general $(\mu, q)$ a measure-kernel pair if $q$ is a Markov kernel and $\mu$ is an invariant probability measure: $\mu q=\mu$. Recall also that the $\Omega$-marginal of a probability measure $\mu$ on some $\boldsymbol{\Omega}_{\ell}$ is denoted by $\mu_{0}$.

Theorem 5.1 Fix a compact subset $\mathcal{U}_{1}$ in the relative interior of $\mathcal{U}$. Then there exists $\beta_{0}>0$ such that, for $\beta \in\left(0, \beta_{0}\right]$ and $\zeta \in \mathcal{U}_{1}$, we can choose $\theta \in$ $\mathbb{R}^{d+1}$ such that the following holds. First $\nabla_{\theta} \lambda(\beta, \theta)=\zeta$ and $\theta$ is a maximizer in (5.3). The martingales $W_{n}^{ \pm}$are uniformly integrable and the pair $\left(\mu^{\theta}, q^{\theta}\right)$ is well-defined by (5.7)-(5.8). We have

$$
\Lambda_{1}(\beta g, \zeta)=-\lambda^{*}(\beta, \zeta) .
$$

A measure-kernel pair $(\mu, q)$ on $\boldsymbol{\Omega}_{1}$ such that $\mu_{0} \ll \mathbb{P}$ satisfies

$$
\Lambda_{1}(\beta g, \zeta)=E^{\mu}[\beta g]-H\left(\mu \times q \mid \mu \times \hat{p}_{1}\right)
$$

if and only if $(\mu, q)=\left(\mu^{\theta}, q^{\theta}\right)$. 
Remark 5.2 Note that even though $\nabla_{\theta} \lambda(\beta, \theta)=\zeta$ does not pick a unique $\theta$, by (5.4) replacing $\theta$ by another maximizer does not change the martingales $W_{n}^{ \pm}$or the pair $\left(\mu^{\theta}, q^{\theta}\right)$. Thus $\zeta$ determines $\left(\mu^{\theta}, q^{\theta}\right)$ uniquely.

Proof The key technical point is to ensure the uniform integrability of the martingales $W_{n}^{ \pm}$. We do so with an $L^{2}$ condition.

Lemma 5.3 Given $C<\infty$ there exists $\beta_{0}=\beta_{0}(C)>0$ such that

$$
\sup _{|\theta| \leq C, \beta \in\left(0, \beta_{0}\right]} \sup _{n} \mathbb{E}\left[\left(W_{n}^{ \pm}\right)^{2}\right]<\infty
$$

Proof We prove Lemma 5.3 for the case of $W_{n}^{+}$. Given $\theta \in \mathbb{R}^{d}$ and $\beta \in(0, c]$, define the tilted kernel

$$
p_{z}^{\theta, \beta}=p_{z} \mathbb{E}\left[e^{\beta g\left(\omega_{0}, z\right)}\right] e^{\theta \cdot z-\lambda(\beta, \theta)},
$$

and let $P^{\theta, \beta}, E^{\theta, \beta}$ denote probabilities and expectations for the random walk that uses the tilted kernel. Let $X_{k}$ and $\widetilde{X}_{k}$ denote two independent walks that use kernel $p_{z}^{\theta, \beta}$ and start at $X_{0}=\widetilde{X}_{0}=0$. Independence of the environments and directedness of the walk give, through a straightforward calculation,

$$
\mathbb{E}\left[\left(W_{n}^{+}\right)^{2}\right]=E^{\theta, \beta}\left[e^{V_{n}}\right] \leq E^{\theta, \beta}\left[\alpha^{L_{n}}\right] \leq E^{\theta, \beta}\left[\alpha^{L_{\infty}}\right]
$$

where

$$
\begin{aligned}
& V_{n}=\sum_{0 \leq k<n} \mathbb{1}\left\{X_{k}=\widetilde{X}_{k}\right\} G\left(Z_{k+1}, \widetilde{Z}_{k+1}\right) \\
& G\left(z, z^{\prime}\right)=\log \frac{\mathbb{E}\left[e^{\beta\left(g\left(\omega_{0}, z\right)+g\left(\omega_{0}, z^{\prime}\right)\right)}\right]}{\mathbb{E}\left[e^{\beta g\left(\omega_{0}, z\right)}\right] \mathbb{E}\left[e^{\beta g\left(\omega_{0}, z^{\prime}\right)}\right]}
\end{aligned}
$$

$L_{n}=\sum_{0 \leq k<n} \mathbb{1}\left\{X_{k}-\widetilde{X}_{k}=0\right\}$ counts the visits to 0 by the symmetric random walk $Y_{k}=X_{k}-\widetilde{X}_{k}$, and

$$
\alpha=\max _{z, z^{\prime} \in \mathcal{R}} e^{G\left(z, z^{\prime}\right)}>1 .
$$

Note that $\alpha \searrow 1$ as $\beta \searrow 0$.

Let $\tau_{0}=0$ and $\tau_{j}=\inf \left\{k>\tau_{j-1}: Y_{k}=0\right\}$ be the times of successive visits to 0 . By the strong Markov property

$$
E^{\theta, \beta}\left[\alpha^{L_{\infty}}\right]=\sum_{k=1}^{\infty} \alpha^{k} P^{\theta, \beta}\left(\tau_{1}<\infty\right)^{k-1} P^{\theta, \beta}\left(\tau_{1}=\infty\right) .
$$

Thus we have $L^{2}$ boundedness in (5.12) if

$$
\alpha P^{\theta, \beta}\left(\tau_{1}<\infty\right) \leq 1-\varepsilon
$$

for some $\varepsilon>0$. The next lemma guarantees that for small enough $\beta, \varepsilon>0$, (5.14) holds simultaneously for all $\theta$ in a given bounded set. This completes the proof of Lemma 5.3. 
Lemma 5.4 For $C<\infty$ there exist $\beta_{0}(C) \in(0, c]$ and $\eta=\eta(C)>0$ such that

$$
\inf _{|\theta| \leq C, \beta \in\left(0, \beta_{0}\right]} P^{\theta, \beta}\left(\tau_{1}=\infty\right) \geq \eta
$$

Proof Let $q_{x}^{\theta, \beta}$ be the kernel of the symmetric walk $Y_{k}$. The support of $q_{x}^{\theta, \beta}$ is the finite set $\mathcal{K}=\mathcal{R}-\mathcal{R}$, a subset of the image of $\mathbb{Z}^{d}$ in the space-time lattice $\mathbb{Z}^{d+1}$. The point about restricting $\theta$ to a bounded set is that then there exists a constant $\delta_{0}>0$ such that $q_{x}^{\theta, \beta} \geq \delta_{0}$ for all $x \in \mathcal{K}$, all $\beta>0$ small enough, and all $\theta$ in the bounded set.

The return probability of the walk is not changed by a linear, isomorphic mapping of the subgroup of $\mathbb{Z}^{d}$ where the walk $Y_{k}$ lives. Thus we may assume that $Y_{k}$ is a symmetric walk in $\mathbb{Z}^{m}$ and aperiodic in the sense of Spitzer [29], namely that the group generated by $\mathcal{K}$ is the entire lattice $\mathbb{Z}^{m}[29$, Sect. 7 , $\mathrm{P} 1]$. It then follows that the characteristic function $\varphi^{\theta, \beta}$ of $q_{x}^{\theta, \beta}$ satisfies $0 \leq$ $\varphi^{\theta, \beta}(t)<1$ for all $t \in[-\pi, \pi]^{m} \backslash\{0\}$.

We bound the return probability with arguments familiar from recurrence considerations [10, Sect. 3.2].

$$
\begin{aligned}
\frac{1}{P^{\theta, \beta}\left(\tau_{1}=\infty\right)} & =\sum_{k=0}^{\infty} P^{\theta, \beta}\left(\tau_{k}<\infty\right)=E^{\theta, \beta}\left[L_{\infty}\right]=\sum_{k=1}^{\infty} P^{\theta, \beta}\left(Y_{k}=0\right) \\
& =(2 \pi)^{-d} \int_{(-\pi, \pi]^{m}} \frac{1}{1-\varphi^{\theta, \beta}(t)} d t
\end{aligned}
$$

The last integral is bounded in two parts. Fix $\delta_{1}>0$ small enough so that $|x|<\pi /\left(3 \delta_{1}\right)$ for all $x \in \mathcal{K}$ and let $B\left(\delta_{1}\right)$ be the open ball of radius $\delta_{1}$ around the origin in $\mathbb{R}^{m}$. For all $t$ in the compact set $[-\pi, \pi]^{m} \backslash B\left(\delta_{1}\right)$, for all $\beta>0$ small enough, and for all $\theta$ in a bounded set we have $\varphi^{\theta, \beta}(t) \leq 1-\varepsilon$ for some fixed $\varepsilon>0$, by continuity.

On the small ball we expand. Let $r \in\left(0, \delta_{1}\right)$ and $|t|=1$.

$$
1-\varphi^{\theta, \beta}(r t)=\sum_{x \in \mathcal{K}}(1-\cos (r t \cdot x)) q_{x}^{\theta, \beta} \geq \frac{r^{2}}{4} \sum_{x \in \mathcal{K}}|t \cdot x|^{2} q_{x}^{\theta, \beta} \geq \delta_{2} r^{2}
$$

for another constant $\delta_{2}>0$. This follows because $q_{x}^{\theta, \beta} \geq \delta_{0}$ and the last expression is a continuous function of $t$ that cannot vanish on the unit sphere because $x \in \mathcal{K}$ generate the entire lattice. Consequently, switching to polar coordinates and since $m \geq 3$ by assumption (5.1),

$$
\int_{B\left(\delta_{1}\right)} \frac{1}{1-\varphi^{\theta, \beta}(t)} d t \leq C \int_{0}^{\delta_{1}} r^{m-1} r^{-2} d r \leq C .
$$

We have shown that the integral in (5.15) is bounded uniformly as $\theta$ varies over a bounded subset of $\mathbb{R}^{d}$ and $\beta>0$ gets small. 
An explicit computation gives

$$
\begin{aligned}
H\left(\mu^{\theta} \times q^{\theta} \mid \mu^{\theta} \times \hat{p}\right) & =E^{\mu^{\theta}}[\beta g]-\lambda(\beta, \theta)+\theta \cdot E^{\mu^{\theta}}\left[Z_{1}\right] \\
& =E^{\mu^{\theta}}[\beta g]-\lambda(\beta, \theta)+\theta \cdot \nabla_{\theta} \lambda(\beta, \theta) \\
& =E^{\mu^{\theta}}[\beta g]+\lambda^{*}\left(\beta, \nabla_{\theta} \lambda(\beta, \theta)\right)
\end{aligned}
$$

One needs to note that the integral $\int \log W_{\infty}^{+} d \mu_{0}^{\theta}$ is well-defined because the integrals $\mathbb{E}\left[W_{\infty}^{+} \log ^{ \pm} W_{\infty}^{+}\right]$are both finite, the $\log ^{+}$one because $W_{\infty}^{+} \in L^{2}(\mathbb{P})$.

The next lemma gives the the point-to-point free energy in the weak disorder regime where quenched quantities agree with their averaged counterparts.

Lemma 5.5 Fix a compact subset $\mathcal{U}_{1}$ in the relative interior of $\mathcal{U}$. Then there exists $\beta_{0}>0$ such that, for $\beta \in\left(0, \beta_{0}\right]$ and $\zeta \in \mathcal{U}_{1}$,

$$
\Lambda_{1}(\beta g, \zeta)=-\lambda^{*}(\beta, \zeta) .
$$

Proof We appeal to the variational formula from (4.3):

$$
-\Lambda_{1}(\beta g, \zeta)=\sup _{t \in \mathbb{R}^{d}}\left\{\zeta \cdot t-\Lambda_{1}\left(\beta g+t \cdot z_{1}\right)\right\}
$$

Since $\zeta \in \mathcal{U}$, the quantity in braces is not changed by projecting $t$ orthogonally to the vector subspace $V$ that is parallel to the affine hull aff $\mathcal{U}$. This is because $t-s \perp V$ implies that $(t-s) \cdot u$ is constant over $u \in$ aff $\mathcal{U}$. So we may restrict the supremum to $t \in V$. Same reasoning gives

$$
\lambda^{*}(\beta, \zeta)=\sup _{t \in V}\{\zeta \cdot t-\lambda(\beta, t)\}
$$

Suppose we can show the existence of $0<\beta_{1}, C<\infty$ such that, for $\beta \in$ $\left(0, \beta_{1}\right]$ and $\zeta \in \mathcal{U}_{1}$,

$$
-\Lambda_{1}(\beta g, \zeta)=\sup _{t \in V:|t| \leq C}\left\{\zeta \cdot t-\Lambda_{1}\left(\beta g+t \cdot z_{1}\right)\right\}
$$

and

$$
\lambda^{*}(\beta, \zeta)=\sup _{t \in V:|t| \leq C}\{\zeta \cdot t-\lambda(\beta, t)\}
$$

Note that (with $t$ in place of $\theta$ in $W_{n}^{+}$)

$$
\begin{aligned}
\Lambda_{1}\left(\beta g+t \cdot z_{1}\right) & =\lim _{n \rightarrow \infty} n^{-1} \log E\left[e^{\beta \sum_{k=0}^{n-1} g\left(\omega_{X_{k}}, Z_{k+1}\right)+t \cdot X_{n}}\right] \\
& =\lim _{n \rightarrow \infty} n^{-1} \log W_{n}^{+}+\lambda(\beta, t) .
\end{aligned}
$$

If we now pick $\beta_{0} \in\left(0, \beta_{1}\right]$ to match $C$ in Lemma 5.3 , the last limit above vanishes for $|t| \leq C$ and $\beta \in\left(0, \beta_{0}\right]$, and (5.19)-(5.20) give

$$
-\Lambda_{1}(\beta g, \zeta)=\sup _{t \in V:|t| \leq C}\{\zeta \cdot t-\lambda(\beta, t)\}=\lambda^{*}(\beta, \zeta) .
$$

This would establish the lemma. 
It remains to show (5.19)-(5.20). From an obvious lower bound and (5.21) with $t=0$

$$
-\Lambda_{1}(\beta g, \zeta) \geq-\Lambda_{1}(\beta g)=-\lambda(\beta, 0) .
$$

Thus (5.19) will follow from the existence of $C<\infty$ such that

$$
\sup _{\beta \in\left(0, \beta_{1}\right]} \sup _{\zeta \in \mathcal{U}_{1}} \sup _{t \in V:|t|>C}\left\{\zeta \cdot t-\Lambda_{1}\left(\beta g+t \cdot z_{1}\right)\right\}<-c_{1} \equiv-\sup _{\beta \in\left(0, \beta_{1}\right]} \lambda(\beta, 0) .
$$

To get a contradiction, suppose $\exists \zeta_{j} \in \mathcal{U}_{1}, \beta_{j} \in\left(0, \beta_{1}\right]$ and $V \ni t_{j} \rightarrow \infty$ such that

$$
\zeta_{j} \cdot t_{j}-\Lambda_{1}\left(\beta_{j} g+t_{j} \cdot z_{1}\right) \geq-c_{1} .
$$

Pass to a subsequence to assume $\zeta_{j} \rightarrow \zeta \in \mathcal{U}_{1}$ and $t_{j} /\left|t_{j}\right| \rightarrow s \in V$.

There exists $z \in \mathcal{R}$ such that $s \cdot(z-\zeta)>0$. If this were not so, we would have $s \cdot z \leq s \cdot \zeta$ for all $z \in \mathcal{R}$, and hence also $s \cdot x \leq s \cdot \zeta$ for all $x \in \mathcal{U}$. But $\zeta \in$ ri $\mathcal{U}$ and $s \in V$ imply that $\zeta+\varepsilon s \in \mathcal{U}$ for small enough $\varepsilon>0$ and we have a contradiction. (This was the point of restricting the suprema to $V$ to begin with.)

Now bound as follows:

$$
\begin{aligned}
& \Lambda_{1}\left(\beta_{j} g+t_{j} \cdot z_{1}\right) \\
& \geq \lim _{n \rightarrow \infty} n^{-1} \log E\left[e^{\beta_{j} \sum_{k=0}^{n-1} g\left(\omega_{X_{k}}, Z_{k+1}\right)+t_{j} \cdot X_{n}}, Z_{1, n}=(z, z, \ldots, z)\right] \\
& =\beta_{j} \mathbb{E}\left[g\left(\omega_{0}, z\right)\right]+t_{j} \cdot z+\log p_{z} .
\end{aligned}
$$

Inequality (5.23) and the above bound give

$$
-\left|t_{j}\right| \frac{t_{j}}{\left|t_{j}\right|} \cdot\left(z-\zeta_{j}\right) \geq \beta_{j} \mathbb{E}\left[g\left(\omega_{0}, z\right)\right]+\log p_{z}-c_{1}
$$

which must eventually fail. We have verified (5.22) and thereby (5.19).

(5.20) comes by essentially the same argument.

We can complete the proof of Theorem 5.1. That (5.11) holds for $(\mu, q)=$ $\left(\mu^{\theta}, q^{\theta}\right)$ comes from (5.16) and (5.17), when $\theta$ is chosen to satisfy $\nabla_{\theta} \lambda(\beta, \theta)=$ $\zeta$.

It remains to prove the uniqueness. Suppose $(\mu, q)$ also satisfies $\mu_{0} \ll \mathbb{P}$ and (5.11). For $0<s<1$ we can create another measure-kernel pair $(\bar{\mu}, \bar{q})$ by $\bar{\mu}=s \mu^{\theta}+(1-s) \mu$ and

$$
\bar{q}\left(\eta, S_{z}^{+} \eta\right)=s \varphi(\eta) q^{\theta}\left(\eta, S_{z}^{+} \eta\right)+(1-s) \psi(\eta) q\left(\eta, S_{z}^{+} \eta\right)
$$

where $\varphi=d \mu^{\theta} / d \bar{\mu}$ and $\psi=d \mu / d \bar{\mu}$. Since $(\bar{\mu}, \bar{q})$ cannot give a larger value on the right side of (5.11), strict convexity of $x \log x$ forces $q\left(\eta, S_{z}^{+} \eta\right)=q^{\theta}\left(\eta, S_{z}^{+} \eta\right)$ $\mu$-a.s. Take a bounded measurable test function $f$. By the stationarity of the $(\mu, q)$-chain

$$
E^{\mu}[f]=E^{\mu}\left[n^{-1} \sum_{k=0}^{n-1} q^{k}(f)\right]=E^{\mu}\left[n^{-1} \sum_{k=0}^{n-1}\left(q^{\theta}\right)^{k}(f)\right] \rightarrow E^{\mu^{\theta}}[f]
$$


where the last limit uses the ergodicity of $\left(\mu^{\theta}, q^{\theta}\right)$ and the absolute continuity $\mu \ll \mu^{\theta}$ which is a consequence of $\mu_{0}^{\theta} \sim \mathbb{P}$. Thus $\mu=\mu^{\theta}$. Theorem 5.1 is proved.

\section{The 1+1 dimensional log-gamma polymer}

This last section of the paper raises a point for future study. One goal is to develop this large deviation approach into a tool for accessing properties of the limiting free energy $\Lambda_{\ell}(g, \zeta)$ even in strong disorder, that is, when $W_{\infty}^{ \pm}$ in (5.5) vanish. We discuss the exactly solvable $1+1$ dimensional log-gamma polymer introduced in [28]. With the help of special information available for this model we show that our formula for $\Lambda(g, \zeta)=\Lambda_{0}(g, \zeta)$ is the same as a known variational formula obtained earlier through a completely different argument. (Note that we are not giving a new independent derivation of this known formula.)

This model is a special case of Example 1.2 with $d=2$ and $\mathcal{R}=\left\{e_{1}, e_{2}\right\}$. So paths started from the origin are nearest-neighbor lattice paths in $\mathbb{Z}_{+}^{2}$ whose steps point in the coordinate directions. The potential is simply $g(\omega)=\omega_{0}$. Coordinates $\omega_{x}$ of the environment are i.i.d. "- $\log$ gamma" distributed. In other words, with a fixed parameter $\rho \in(0, \infty)$, the variable $e^{-\omega_{x}}$ has $\operatorname{Gamma}(\rho)$ distribution: for $t \in \mathbb{R}$,

$$
\mathbb{P}\left\{\omega_{x} \leq t\right\}=\mathbb{P}\left\{e^{-\omega_{x}} \geq e^{-t}\right\}=\Gamma(\rho)^{-1} \int_{e^{-t}}^{\infty} s^{\rho-1} e^{-s} d s .
$$

In the log-gamma case the limit $\Lambda(g, \zeta)$ can be computed by introducing a stationary version of the model and by doing essentially a "hydrodynamic limit" computation. For $\zeta=(x, 1-x) \in \mathbb{R}_{+}^{2}$ this leads to

$$
\Lambda(g,(x, 1-x))=-\log 2+\inf _{\theta \in(0, \rho)}\left\{-x \Psi_{0}(\theta)-(1-x) \Psi_{0}(\rho-\theta)\right\}
$$

where $\Psi_{0}(t)=\Gamma^{\prime}(t) / \Gamma(t)$ is the digamma function. (See Theorem 2.1 and the proof of Lemma 4.1 in [14].)

Recall from Remark 4.2 the duality

$$
I^{g}(\zeta)=\sup _{t \in \mathbb{R}^{2}}\{\zeta \cdot t-\sigma(t)\}
$$

for the level 1 rate function $I^{g}$ of Theorem 4.1, where $\sigma$ is the quenched limiting logarithmic moment generating function

$$
\sigma(t)=\lim _{n \rightarrow \infty} n^{-1} \log E^{Q_{n}^{g, \omega}}\left(e^{t \cdot X_{n}}\right) .
$$

Ideally we would like to derive (6.1) from the theory developed in this paper. This is left for future work. By utilizing (6.1) itself, we can make the following observation.

Remark 6.1 In the log-gamma model, formula (6.2) specializes to (6.1). 
Before proving Remark 6.1, let us explain its point. Derivations of (6.1) ([14] and also [28]) do not involve large deviations. Instead (6.1) is the minimal free energy of a stationary log-gamma polymer that is compatible with the choice of parameter $\rho$ for the bulk weights. These stationary models are indexed by $\theta \in(0, \rho)$ which parametrizes their initial, or boundary, conditions. The existence of a stationary version is a special feature of the log-gamma model.

Because rate function $I^{g}(\zeta)$ comes from a contraction from a higher level LDP, variational formula (6.2) should be explainable through finding a natural Markov process on environments and paths under which the path has speed $\zeta$. (This is exactly what was done in Theorem 5.1 in the $L^{2}$ weak disorder regime.) Consequently, (6.1) should also represent the choice of a natural Markov process, indexed by the parameter $\theta$. Thus the Markov processes singled out by variational formulas could be the general analogue of the special stationary boundary conditions of the log-gamma model. If the choice of these Markov processes were understood, some progress might be possible in polymers in strong disorder beyond the exactly solvable log-gamma model.

Proof of Remark 6.1 On $(0, \infty), \Psi_{0}$ is strictly concave, strictly increasing and $\Psi_{0}(0+)=-\infty$. The trigamma function $\Psi_{1}=\Psi_{0}^{\prime}$ is strictly convex, strictly decreasing, $\Psi_{1}(0+)=\infty$ and $\Psi_{1}(\infty)=0$. Let $\theta(x) \in(0, \rho)$ be uniquely determined by

$$
x \Psi_{1}(\theta(x))-(1-x) \Psi_{1}(\rho-\theta(x))=0 .
$$

It minimizes in (6.1) so that

$$
\Lambda(g,(x, 1-x))=-\log 2-x \Psi_{0}(\theta(x))-(1-x) \Psi_{0}(\rho-\theta(x)) .
$$

First we reduce the dimension in $(6.2)$. Note that $(1,1) \cdot X_{n}=n$, and so $\sigma\left(t_{1}+b, t_{2}+b\right)=\sigma\left(t_{1}, t_{2}\right)+b$. Then for $\zeta=(x, 1-x), x \in[0,1],(6.2)$ simplifies to

$$
I^{g}(x, 1-x)=\sup _{t \in \mathbb{R}}\{x t-\sigma(t, 0)\} .
$$

As $n \rightarrow \infty$,

$$
\begin{aligned}
& n^{-1} \log E^{Q_{n}^{g, \omega}}\left(e^{\left(t_{1}, t_{2}\right) \cdot X_{n}}\right) \\
& =n^{-1} \log \sum_{k=0}^{n} e^{\left(t_{1}, t_{2}\right) \cdot(k, n-k)} E\left[e^{\sum_{k=0}^{n-1} \omega_{X_{k}}}, X_{n}=(k, n-k)\right] \\
& -n^{-1} \log E\left[e^{\sum_{k=0}^{n-1} \omega_{X_{k}}}\right] \\
& \rightarrow \sup _{x \in[0,1]}\left\{\left(t_{1}, t_{2}\right) \cdot(x, 1-x)+\Lambda(g,(x, 1-x))-\Lambda(g)\right\} .
\end{aligned}
$$

Consequently

$$
\begin{aligned}
\sigma(t, 0) & =\sup _{x \in[0,1]}\left\{t x-x \Psi_{0}(\theta(x))-(1-x) \Psi_{0}(\rho-\theta(x))\right\}-\log 2-\Lambda(g) \\
& =t x-x \Psi_{0}(\theta(x))-(1-x) \Psi_{0}(\rho-\theta(x))-\log 2-\Lambda(g)
\end{aligned}
$$


for the unique $x$ determined by

$$
t=\Psi_{0}(\theta(x))-\Psi_{0}(\rho-\theta(x)) .
$$

Change variables between $t \in \mathbb{R}$ and $\theta \in(0, \rho)$ via equation

$$
t=\Psi_{0}(\theta)-\Psi_{0}(\rho-\theta) .
$$

Then

$$
\sigma\left(\Psi_{0}(\theta)-\Psi_{0}(\rho-\theta), 0\right)=-\Psi_{0}(\rho-\theta)-\log 2-\Lambda(g) .
$$

To obtain (6.1) from (6.3), perform the change of variable (6.4) on the right, while on the left use $I^{g}(x, 1-x)=\Lambda(g)-\Lambda(g,(x, 1-x))$.

\section{A A convex analysis lemma}

Lemma A.1 Let $\mathcal{I}$ be a finite subset of $\mathbb{R}^{d}$ and $\zeta \in \operatorname{co} \mathcal{I}$. Suppose $\zeta=\sum_{z \in \mathcal{I}} \beta_{z} z$ with each $\beta_{z}>0$ and $\sum_{z \in \mathcal{I}} \beta_{z}=1$. Let $\xi_{n} \in \operatorname{co} \mathcal{I}$ be a sequence such that $\xi_{n} \rightarrow \zeta$. Then there exist coefficients $\alpha_{z}^{n} \geq 0$ such that $\sum_{z \in \mathcal{I}} \alpha_{z}^{n}=1, \xi_{n}=\sum_{z \in \mathcal{I}} \alpha_{z}^{n} z$ and for each $z \in \mathcal{I}, \alpha_{z}^{n} \rightarrow \beta_{z}$ as $n \rightarrow \infty$.

Furthermore, assume $\mathcal{I} \subset \mathbb{Q}^{d}$ and $\xi_{n} \in \mathbb{Q}^{d}$. Then the coefficients $\alpha_{z}^{n}$ can be taken rational.

Proof First we reduce the proof to the case where there exists a subset $\mathcal{I}_{0} \subset \mathcal{I}$ such that $\mathcal{I}_{0}$ is affinely independent and generates the same affine hull as $\mathcal{I}$, and $\xi_{n} \in \operatorname{co} \mathcal{I}_{0}$ for all $n$. To justify this reduction, note that there are finitely many such sets $\mathcal{I}_{0}$, and each $\xi_{n}$ must lie in the convex hull of some $\mathcal{I}_{0}$ (Carathéodory's Theorem [27, Theorem 17.1] applied to the affine hull of $\mathcal{I}$ ). All but finitely many of the $\xi_{n}$ 's are contained in subsequences that lie in a particular co $\mathcal{I}_{0}$. The coefficients of the finitely many remaining $\xi_{n}$ 's are irrelevant for the claim made in the lemma.

After the above reduction, the limit $\xi_{n} \rightarrow \zeta$ forces $\zeta \in \operatorname{co} \mathcal{I}_{0}$. The points $\tilde{z} \in \mathcal{I} \backslash \mathcal{I}_{0}$ lie in the affine hull of $\mathcal{I}_{0}$ and hence have barycentric coordinates:

$$
\gamma_{z, \tilde{z}} \in \mathbb{R}, \quad \tilde{z}=\sum_{z \in \mathcal{I}_{0}} \gamma_{z, \tilde{z} z} z, \quad \sum_{z \in \mathcal{I}_{0}} \gamma_{z, \tilde{z}}=1 \quad \text { for } \quad \tilde{z} \in \mathcal{I} \backslash \mathcal{I}_{0} .
$$

Consequently

$$
\zeta=\sum_{z \in \mathcal{I}} \beta_{z} z=\sum_{z \in \mathcal{I}_{0}}\left(\beta_{z}+\sum_{\tilde{z} \in \mathcal{I} \backslash \mathcal{I}_{0}} \gamma_{z, z} \beta_{\tilde{z}}\right) z \equiv \sum_{z \in \mathcal{I}_{0}} \bar{\beta}_{z} z
$$

where the last identity defines the unique barycentric coordinates $\bar{\beta}_{z}$ of $\zeta$ relative to $\mathcal{I}_{0}$. Define the $\mathcal{I}_{0} \times \mathcal{I}$ matrix $A=\left[I \mid\left\{\gamma_{z, z}\right\}\right]$ where $I$ is the $\mathcal{I}_{0} \times \mathcal{I}_{0}$ identity matrix and $(z, \tilde{z})$ ranges over $\mathcal{I}_{0} \times\left(\mathcal{I} \backslash \mathcal{I}_{0}\right)$. Then (A.1) is the identity $A \beta=\bar{\beta}$ for the (column) vectors $\beta=\left(\beta_{z}\right)_{z \in \mathcal{I}}$ and $\bar{\beta}=\left(\bar{\beta}_{z}\right)_{z \in \mathcal{I}_{0}}$. Since $\eta=[\bar{\beta} 0]^{t}$ is also a solution of $A \eta=\bar{\beta}$, we can write $\beta=[\bar{\beta} 0]^{t}+y$ with $y \in \operatorname{ker} A$.

Let $\xi_{n}=\sum_{z \in \mathcal{I}_{0}} \bar{\alpha}_{z}^{n} z$ define the barycentric coordinates of $\xi_{n}$. Since the coordinates are unique, $\xi_{n} \rightarrow \zeta$ forces $\bar{\alpha}^{n} \rightarrow \bar{\beta}$. Let $\alpha^{n}=\left[\bar{\alpha}^{n} 0\right]^{t}+y$. Then $A \alpha^{n}=\bar{\alpha}^{n}$ which says that $\xi_{n}=\sum_{z \in \mathcal{I}} \alpha_{z}^{n} z$. Also $\alpha^{n} \rightarrow \beta$. Since $\beta_{z}>0$, inequality $\alpha_{z}^{n} \geq 0$ fails at most finitely many times, and for finitely many $\xi_{n}$ we can replace the $\alpha_{z}^{n}$ 's with any coefficients that exist by $\xi_{n} \in \operatorname{co} \mathcal{I}$. Lastly, for $\sum_{z \in \mathcal{I}} \alpha_{z}^{n}=1$ we need $\sum_{z \in \mathcal{I}} y_{z}=0$. This comes from $A y=0$ because the column sums of $A$ are all 1 . This completes the proof of the first part of the lemma.

Assume now that $\mathcal{I} \subset \mathbb{Q}^{d}$ and $\xi_{n} \in \mathbb{Q}^{d}$. Then by Lemma A.1. in [26] the vector $\bar{\alpha}^{n}$ is rational. By Lemma A.2. in [23] we can find rational vectors $y^{n} \in \operatorname{ker} A$ such that $y^{n} \rightarrow y$. This time take $\alpha^{n}=\left[\bar{\alpha}^{n} 0\right]^{t}+y^{n}$. 


\section{B A concentration inequality}

We state a concentration inequality for the case of a bounded potential $\mathrm{g}$. It comes from the ideas of Liu and Watbled [18], in the form given by Comets and Yoshida [5].

Lemma B.1 Let $\mathbb{P}$ be an i.i.d. product measure on a product space $\Omega=\Gamma^{\mathbb{Z}^{d}}$ with generic elements $\omega=\left(\omega_{x}\right)_{x \in \mathbb{Z}^{d}}$. Let $g: \boldsymbol{\Omega}_{\ell} \rightarrow \mathbb{R}$ be a bounded measurable function such that, for each $z_{1, \ell} \in \mathcal{R}^{\ell}, g\left(\cdot, z_{1, \ell}\right)$ is a local function of $\omega$. Let $\zeta \in \mathcal{U}$ and

$$
F_{n}(\omega)=\log E\left[e^{\sum_{k=0}^{n-1} g\left(T_{X_{k}} \omega, Z_{k+1, k+\ell}\right)} \mathbb{1}\left\{X_{n}=\hat{x}_{n}(\zeta)\right\}\right] .
$$

Let $\mathcal{U}_{0}$ be a face of $\mathcal{U}$ such that $\zeta \in \mathcal{U}_{0}$, and assume that $0 \notin \mathcal{U}_{0}$.

Then there exist constants $B, c \in(0, \infty)$ such that, for all $n \in \mathbb{N}$ and $\varepsilon \in(0, c)$,

$$
\mathbb{P}\left\{\omega:\left|F_{n}(\omega)-n \Lambda_{\ell}(g, \zeta)\right| \geq n \varepsilon\right\} \leq 2 e^{-B \varepsilon^{2} n} .
$$

Proof Since $n^{-1} \mathbb{E} F_{n} \rightarrow \Lambda_{\ell}(g, \zeta)$, we can prove instead

$$
\mathbb{P}\left\{\omega:\left|F_{n}(\omega)-\mathbb{E} F_{n}\right| \geq n \varepsilon\right\} \leq 2 e^{-B \varepsilon^{2} n} .
$$

As before, with $\mathcal{R}_{0}=\mathcal{R} \cap \mathcal{U}_{0}$ we have $\mathcal{U}_{0}=$ co $\mathcal{R}_{0}$, any admissible path $x_{0, n}$ with $x_{n}=\hat{x}_{n}(\zeta)$ uses only $\mathcal{R}_{0}$-steps, and from $0 \notin \mathcal{U}_{0}$ follows the existence of $\hat{u} \in \mathbb{Z}^{d}$ such that $\hat{u} \cdot z \geq 1$ for all $z \in \mathcal{R}_{0}$. Set $M_{0}=\max _{z \in \mathcal{R}_{0}} \hat{u} \cdot z$.

Fix $r_{0} \in \mathbb{N}$ so that $g\left(\omega, z_{1, \ell}\right)$ depends on $\omega$ only through $\left\{\omega_{x}:|x \cdot \hat{u}|<r_{0}\right\}$. Let $n_{0} \in \mathbb{N}$ be such that $n_{0} r_{0} \geq M_{0} n+r_{0}$. On $\Omega$ define the filtration $\mathcal{H}_{0}=\{\emptyset, \Omega\}, \mathcal{H}_{j}=\sigma\left\{\omega_{x}: x \cdot \hat{u} \leq j r_{0}\right\}$ for $1 \leq j \leq n_{0}$. Since $x_{n} \cdot \hat{u} \leq M_{0} n, F_{n}$ is $\mathcal{H}_{n_{0}}$-measurable.

To apply Lemma A.1 of $[5]$ we need to find $G_{1}, \ldots, G_{n_{0}} \in L^{1}(\mathbb{P})$ such that

$$
\mathbb{E}\left[G_{j} \mid \mathcal{H}_{j-1}\right]=\mathbb{E}\left[G_{j} \mid \mathcal{H}_{j}\right]
$$

and

$$
\mathbb{E}\left[e^{t\left|F_{n}-G_{j}\right|} \mid \mathcal{H}_{j-1}\right] \leq b
$$

for constants $t, b \in(0, \infty)$ and all $1 \leq j \leq n_{0}$.

For the background random walk define stopping times

$$
\rho_{j}=\inf \left\{k \geq 0: x_{k} \cdot \hat{u} \geq(j-2) r_{0}\right\}
$$

and

$$
\sigma_{j}=\inf \left\{k \geq 0: x_{k} \cdot \hat{u} \geq(j+1) r_{0}\right\} .
$$

Abbreviate $\varphi(x)=\mathbb{1}\left\{x=\hat{x}_{n}(\zeta)\right\}$. For $1 \leq j \leq n_{0}$ put

$$
W_{j}=\exp \left\{\sum_{\substack{k: 0 \leq k<n \wedge \rho_{j} \\ n \wedge \sigma_{j} \leq k<n}} g\left(T_{x_{k}} \omega, z_{k+1, k+\ell}\right)\right\}
$$

and

$$
G_{j}(\omega)=\log E\left[W_{j} \varphi\left(X_{n}\right)\right] .
$$

Then $W_{j}$ does not depend on $\left\{\omega_{x}:(j-1) r_{0} \leq x \cdot \hat{u} \leq j r_{0}\right\}$ and consequently (B.4) holds by the independence of the $\left\{\omega_{x}\right\}$.

Let $t \in \mathbb{R} \backslash(0,1)$. By Jensen's inequality,

$$
\begin{aligned}
e^{t\left(F_{n}-G_{j}\right)} & =\left(\frac{E\left[W_{j} e^{\sum_{k=n \wedge \rho_{j}}^{n \wedge \sigma_{j}-1} g\left(T_{X_{k}} \omega, Z_{k+1, k+\ell}\right)} \varphi\left(X_{n}\right)\right]}{E\left[W_{j} \varphi\left(X_{n}\right)\right]}\right)^{t} \\
& \leq \frac{E\left[W_{j} e^{t \sum_{k=n \wedge \rho_{j}}^{n \wedge \sigma_{j}-1} g\left(T_{X_{k}} \omega, Z_{k+1, k+\ell}\right)} \varphi\left(X_{n}\right)\right]}{E\left[W_{j} \varphi\left(X_{n}\right)\right]} \\
& \leq \frac{E\left[W_{j} e^{C|t|\left(\sigma_{j}-\rho_{j}\right)} \varphi\left(X_{n}\right)\right]}{E\left[W_{j} \varphi\left(X_{n}\right)\right]} \leq e^{C|t|}
\end{aligned}
$$


since $g$ is bounded and $\sigma_{j}-\rho_{j} \leq 3 r_{0}$. This implies (B.5) since $t$ can be taken of either sign.

Lemma A.1 of [5] now gives (B.2). Note that parameter $n$ in Lemma A.1 of [5] is actually our $n_{0}$. But the ratio $n / n_{0}$ is bounded and bounded away from zero so this discrepancy does not harm (B.3).

Acknowledgements F. Rassoul-Agha's work was partially supported by NSF Grant DMS0747758. T. Seppäläinen's work was partially supported by NSF Grant DMS-1003651 and by the Wisconsin Alumni Research Foundation.

\section{References}

1. Bolthausen, E., Sznitman, A.S.: Ten lectures on random media, DMV Seminar, vol. 32. Birkhäuser Verlag, Basel (2002)

2. Carmona, P., Hu, Y.: Fluctuation exponents and large deviations for directed polymers in a random environment. Stochastic Process. Appl. 112(2), 285-308 (2004)

3. Comets, F., Shiga, T., Yoshida, N.: Probabilistic analysis of directed polymers in a random environment: a review. In: Stochastic analysis on large scale interacting systems, Adv. Stud. Pure Math., vol. 39, pp. 115-142. Math. Soc. Japan, Tokyo (2004)

4. Comets, F., Yoshida, N.: Directed polymers in random environment are diffusive at weak disorder. Ann. Probab. 34(5), 1746-1770 (2006)

5. Comets, F., Yoshida, N.: Branching random walks in space-time random environment: Survival probability, global and local growth rates. Journal of Theoretical Probability 24, 657-687 (2011)

6. Corwin, I.: The Kardar-Parisi-Zhang equation and universality class (2011). URL http://arxiv.org/abs/1106.1596. Preprint

7. Corwin, I., O'Connell, N., Seppäläinen, T., Zygouras, N.: Tropical combinatorics and Whittaker functions (2011). URL http://arxiv.org/abs/1110.3489. Preprint

8. Cox, J.T., Gandolfi, A., Griffin, P.S., Kesten, H.: Greedy lattice animals. I. Upper bounds. Ann. Appl. Probab. 3(4), 1151-1169 (1993)

9. Dembo, A., Zeitouni, O.: Large deviations techniques and applications, Applications of Mathematics (New York), vol. 38, second edn. Springer-Verlag, New York (1998)

10. Durrett, R.: Probability: theory and examples, second edn. Duxbury Press, Belmont, CA (1996)

11. Ekeland, I., Témam, R.: Convex analysis and variational problems, Classics in Applied Mathematics, vol. 28, english edn. Society for Industrial and Applied Mathematics (SIAM), Philadelphia, PA (1999). Translated from the French

12. Gandolfi, A., Kesten, H.: Greedy lattice animals. II. Linear growth. Ann. Appl. Probab. 4(1), 76-107 (1994)

13. Georgiou, N.: Positive and zero temperature polymer models (2011). Thesis (Ph.D.)University of Madison-Wisconsin

14. Georgiou, N., Seppäläinen, T.: Large deviation rate functions for the partition function in a log-gamma distributed random potential (2011). URL http://arxiv.org/abs/1110.3544. Preprint

15. den Hollander, F.: Random polymers, Lecture Notes in Mathematics, vol. 1974. Springer-Verlag, Berlin (2009). Lectures from the 37th Probability Summer School held in Saint-Flour, 2007

16. Kassay, G.: A simple proof for König's minimax theorem. Acta Math. Hungar. 63(4), 371-374 (1994)

17. Liggett, T.M.: Interacting particle systems. Classics in Mathematics. Springer-Verlag, Berlin (2005). Reprint of the 1985 original

18. Liu, Q., Watbled, F.: Exponential inequalities for martingales and asymptotic properties of the free energy of directed polymers in a random environment. Stochastic Process. Appl. 119(10), 3101-3132 (2009)

19. Martin, J.B.: Linear growth for greedy lattice animals. Stochastic Process. Appl. 98(1), 43-66 (2002) 
20. Moreno Flores, G.R.: Asymmetric directed polymers in random environments (2010). URL http: //arxiv.org/abs/1009.5576. Preprint

21. Mourrat, J.C.: Lyapunov exponents, shape theorems and large deviations for the random walk in random potential (2011). URL http://arxiv.org/abs/1106.3995. Preprint

22. Piza, M.S.T.: Directed polymers in a random environment: some results on fluctuations. J. Statist. Phys. 89(3-4), 581-603 (1997). DOI 10.1007/BF02765537

23. Rassoul-Agha, F., Seppäläinen, T.: Quenched invariance principle for multidimensional ballistic random walk in a random environment with a forbidden direction. Ann. Probab. 35(1), 1-31 (2007)

24. Rassoul-Agha, F., Seppäläinen, T.: A course on large deviation theory with an introduction to Gibbs measures (2010). URL http://www.math.utah.edu/ firas/Papers/rassoul-seppalainen-ldp.pdf . Preprint

25. Rassoul-Agha, F., Seppäläinen, T.: Process-level quenched large deviations for random walk in random environment. Ann. Inst. H. Poincaré Probab. Statist. 47(1), 214-242 (2011)

26. Rassoul-Agha, F., Seppäläinen, T., Yilmaz, A.: Quenched large deviations for random walks in random environments and random potentials. Comm. Pure Appl. Math. (2012). URL http://www .math.utah.edu/ firas/Papers/. To appear.

27. Rockafellar, R.T.: Convex analysis. Princeton Mathematical Series, No. 28. Princeton University Press, Princeton, N.J. (1970)

28. Seppäläinen, T.: Scaling for a one-dimensional directed polymer with boundary conditions. Ann. Probab. 40(1), 19-73 (2012)

29. Spitzer, F.: Principles of random walks, second edn. Springer-Verlag, New York (1976). Graduate Texts in Mathematics, Vol. 34

30. Sznitman, A.S.: Shape theorem, Lyapounov exponents, and large deviations for Brownian motion in a Poissonian potential. Comm. Pure Appl. Math. 47(12), 1655-1688 (1994)

31. Sznitman, A.S.: Brownian motion, obstacles and random media. Springer Monographs in Mathematics. Springer-Verlag, Berlin (1998)

32. Sznitman, A.S.: Topics in random walks in random environment. In: School and Conference on Probability Theory, ICTP Lect. Notes, XVII, pp. 203-266 (electronic). Abdus Salam Int. Cent. Theoret. Phys., Trieste (2004)

33. Varadhan, S.R.S.: Large deviations and entropy. In: Entropy, Princeton Ser. Appl. Math., pp. 199-214. Princeton Univ. Press, Princeton, NJ (2003)

34. Yilmaz, A.: Large deviations for random walk in a space-time product environment. Ann. Probab. 37(1), 189-205 (2009)

35. Zeitouni, O.: Random walks in random environment. In: Lectures on probability theory and statistics, Lecture Notes in Math., vol. 1837, pp. 189-312. Springer, Berlin (2004)

36. Zerner, M.P.W.: Directional decay of the Green's function for a random nonnegative potential on $\mathbf{Z}^{d}$. Ann. Appl. Probab. 8(1), 246-280 (1998) 